\title{
Nonlinear elasto-plastic model for dense granular flow
}

\author{
Ken Kamrin \\ Harvard School of Engineering and Applied Sciences, 29 Oxford St, Cambridge, MA 02139
}

\begin{abstract}
This work proposes a model for granular deformation that predicts the stress and velocity profiles in well-developed dense granular flows. Recent models for granular elasticity (Jiang and Liu 2003) and rate-sensitive fluid-like flow (Jop et al. 2006) are reformulated and combined into one universal elasto-plastic law, capable of predicting flowing regions and stagnant zones simultaneously in any arbitrary 3D flow geometry. The unification is performed by justifying and implementing a KrönerLee decomposition, with care taken to ensure certain continuum physical principles are necessarily upheld. The model is then numerically implemented in multiple geometries and results are compared to experiments and discrete simulations.
\end{abstract}

\section{Introduction}

A general constitutive law for granular materials, valid over all common regimes of deformation, remains an important open problem for several communities. Mechanicians and materials engineers have modeled granular materials for centuries guided by principles of continuum solid mechanics [63, 81, 39, 79, 1, 78, 47, 82], where various failure and yield postulates have been employed. In recent years, a resurgence of interest in the topic has arisen among physicists [41, 18, 45, 24, 36, 5], where modeling efforts have been directed primarily toward statistical and fluid dynamical approaches.

Some of the difficulty in modeling dense granular materials is due to the existence of highly disparate responses when material is flowing "slowly" or "moderately" (see section 4.2), both of which only vaguely relate to the completely static response. To describe certain phenomenological behaviors, an astounding array of physical hypotheses have been suggested. These include: rolling and static phase interactions 12, 13, 15, 14, Bagnold rheology generated by "granular eddies" 25], granular temperature-dependent viscosity [78], density-dependent viscosity 57, 10], non-local stress propagation [60, 11], free-volume diffusion opposing gravity [55, 62, 64, 8, 75], "shear transformation zones" coupled to free-volume kinetics [54, 53], and partial fluidization governed by a GinzbergLandau order parameter 3, 4]. Such theories can typically fit certain experimental data 59, usually for a specific geometry: avalanching surface flows [12, 13, 15, 14, 3], inclined planes [6, 25], Couette cells [57, 10], inclined chutes [70, 69], or wide silo [55, 62, 64, 8, 75]. A good review of granular flow theories can be found in [7].

Some models have been developed that are general enough for multiple flow environments 1,79 , 47, 4]. In particular, the work of Jop et al. 44 introduces and tests a generic, 3D, non-Newtonian fluid constitutive law representing "moderate" sized granular shear-rate as a local function of the Cauchy stress. The material is treated as a Bingham fluid and a computation of the stress and flow profiles can be made in regions above a Drucker-Prager yield criterion. While a breakthrough by many standards, the law makes rigid-body assumptions in static phases where stresses are not computed. In many circumstances, granular assemblies can establish static zones at walls (such as

Email addresses: kkamrin@seas.harvard.edu (Ken Kamrin)

URL: http://people.seas.harvard.edu/ kkamrin/ (Ken Kamrin) 
in flowing hoppers and silos [63, 81]), and effective flow computation is impingent on the ability to model traction-based interaction laws between sub-yield material and the system boundaries. Stress-based quantities in sub-yield zones are needed to approach a variety of open questions in granular flow theory, such as how "kick-stresses" [63, 21] develop in draining storage bins.

This work seeks a general constitutive law for 3D, dense flowing granular materials. Specifically, we focus on flows with significant regions of moderate shearing, as would be expected in day-today environments like flowing chutes, hoppers, and heaps. To this end, the model of Jop et al. is integrated with an appropriate granular solid response, by way of a joint elasto-plastic treatment. The unified formulation can be used to predict the stress and flow profiles everywhere, for an arbitrary geometry with arbitrary admissible kinematic/traction boundary conditions. For sufficient generality and accuracy in describing the static phase, we ultimately choose to pair with the granular elasticity law proposed recently by Jiang and Liu [42]. This happens to be a natural choice for several reasons, not the least of which is its proven capability to represent several static and acoustic phenomena observed in granular matter.

The process of constructing a combined law is itself non-trivial. Both components require some degree of reinterpretation - the Jop et al. law is converted to an evolution law for the plastic deformation gradient, and the Jiang-Liu elasticity law is modified to permit flowing states while maintaining elastic deformation. Moreover, some essential postulates of the chosen elasto-plastic framework are not obvious in well-developed flow, requiring additional substantiation.

This paper is organized as follows. First, we describe some general requirements for a continuum treatment of granular statics/flow (section 2). Then a discussion of past solid and flow constitutive laws for dense granular media are reviewed (sections 3 and (4), ultimately culminating in a description of the two laws mentioned above. The process of combining these responses is discussed (section 5), which ends with a summary of equations for the proposed model. Finally, section 6 provides discussion on the numerical implementation of these equations and displays results in several flow geometries, with comparisons against the data of [44],[59], and [76].

\section{Granular matter as a continuum}

Before development ensues, we take a moment to lay out the limits of validity for a first-order continuum treatment of a discrete granular collection, as shall be proposed herein. For a more detailed set of arguments on deterministic limits in random composite materials, the reader is directed to the seminal work of Ostoja-Starzewski [67 66].

Recent Discrete Element Method (DEM) simulations of 76 . have shown that in multiple 3D welldeveloped flow environments, a dense granular element of width $\sim 5 d$ (for $d=$ particle diameter) captures many of the plastic flow properties expected of an RVE. Among neighboring volume elements of this width, the average stress and deformation rate appear to vary smoothly. Within a flowing element, the eigenvectors of the instantaneous space-average Cauchy stress align to a high degree with those of the deformation rate tensor, evidencing the onset of a deterministic relationship between the two fields. Moreover, a predictable dependence of the packing fraction on the pressure and shearing rate emerges at this length-scale. Of course, the representative element width is not a universal constant; environments with large spatial gradients necessitate smaller elements. Thus the $5 d$ element width can be interpreted as a common limit for day-to-day flows, compacted primarily by gravity, where boundary conditions on the global body vary on a scale larger than $O(10 d)$.

The result is not outlandish in light of the past observations by Glasser and Goldhirsch [29] and Goldenberg et al. 31], where in depth 2D studies on disk arrays were performed to quantify the effects of spatial averaging on granular stresses. Rycroft's result is in line with the extremal trends observed over the spectrum from rapid/dilute disk flows to static/elastic deformation of a disk assembly. Furthermore, it is found that static materials gain resolution independence on a similar scale to flowing materials, suggesting that a $5 d$ element width could give valid RVE behavior for dense granular materials in either static or flowing states. 
Previous arguments against a granular continuum, particularly in the static phase but also during flow, have focused on the presence of force chains in granular matter. In 2D experiments and simulations of disk assemblies [9, 28], concentrated chains of interparticle force have been shown to exist over many particle widths. It was argued that if forces are not homogeneous at a meso-scale, continuum relations at this scale cannot exist. However, in 3D simulations of flowing monodisperse sphere packings, we observe that the force chains have a dramatically shorter length 76]. A possible geometric explanation for this phenomenon is that a $3 \mathrm{D}$ granular assembly has a much higher average coordination number, reducing the likelihood that only two contacts maintain the majority of the force on one grain. These simulations also include interparticle contact friction, which may contribute to the smoothing out of force chains as has been previously shown in 2D static disk assemblies [32]. Typically speaking, whether flowing or static, we observe that a $5 d$ granular volume element contains a diffuse network of contact forces, enabling a sufficient degree homogenization of the stresses at this scale.

Frequently, a granular collection that has been set into motion will possess regions of fluid-like flow adjacent to essentially solid-like regions. Examples of such flows include wide draining silos [16, 77] and hoppers (so-called "core flow") where a broad column of material extending upward from the orifice flows like a fluid, while regions closer to the side walls remain almost completely static. As will be discussed later, perfectly clear solid/fluid interfaces are rarely observed in granular flow, which has led some to believe that the solid-like zone is actually just a 'highly viscous' fluid region [78, 57, 10]. Solid-like material does undergo intermittent rearrangement events when close to a zone of moderate flow-rate, but we find that the stresses in these regions have essentially zero rate-dependence. For example, when a DEM simulation of silo flow is suddenly arrested, say by shutting the orifice, the stresses in the solid-like regions remain virtually unchanged, supporting static shear stress like a solid. It appears that a mechanism disconnected from the flow-rate or any notion of viscosity is responsible for maintaining the stress tensor in solid-like granular matter, even if occasional failure events are occurring within. This suggests that a continuum description for granular statics is likely to be disjointed from the flow description. Hence, we seek a general model that splices separate statics and flow laws into one constitutive form.

\section{Continuum statics}

\subsection{Stress-only laws vs. elasticity}

To close the equations for static force balance, two approaches have been utilized: stress-only laws and elasticity. Stress-only relationships constrain the stresses directly, by asserting that the stress tensor must satisfy some a priori relationship. Examples include: Janssen's law of differential slices (originally proposed by Janssen in 1895) where vertical and horizontal stresses are set to be proportional, limit-state Mohr-Coulomb plasticity or the "Incipient Failure Everywhere" (IFE) hypothesis [81] where a Mohr-Coulomb failure line is required to exist at all locations, and "Oriented Stress Linearity" [1] where stresses propagate in directions aligned with the presumptive microstructure of the packing.

While stress-only relationships are convenient and have had some success, their physical assumptions can be questionable. For example, static granular matter is rarely in a limit-state of incipient failure [76] and wall shear is not compatible with a Janssen-style stress tensor [63]. Most stress-only laws are defined only for 2D media, which brings out issues of generality. They often predict a "hyperbolic" character to the stress profile, where stress quantities propagate in certain directions from the boundaries. The notion of force propagation was backed chiefly by the observation of a double-peak pressure distribution beneath a bed of grains undergoing a point force from the top. Work by Goldenberg and Goldhirsch [32] has shown, however, that in the presence of interparticle friction and a large system size to particle size ratio (as commonly found in engineering applications) the pressure distribution is indeed a broad single peak beneath the point force, as one would expect for an elastic bulk media. 
This brings us to elasticity, which shall be our preferred method for granular statics. It is a sensible approach seeing as the grains themselves are elastic bodies presumably enabling generalization to an Effective Medium Theory (EMT) where grain-level elasticity extends statistically to a continuum mean-field theory [22, 20, 89, 65]. Reversible (elastic) deformations have been observed in granular matter for strains less than $10^{-4}$ [51]. This is negligibly small compared to the size of typical plastic deformation. However, grains are commonly composed of stiff material indicating the important role that small elastic strains may play in the development of the stress profile. It could be argued that the barely noticeable elastic strain of a static assembly is what originally impelled scientists to seek stress-only laws, so that rigid-body assumptions could be used. Rather, it seems there is no generally applicable stress-only constraint to accurately define a 3D stress tensor. The elastic strains are small but non-ignorable, and bear essential importance to the stress description.

Bulk elasticity for cohesionless grains is not likely to have a simple form since, for example, the material is unable to support tension and thus the small strain response cannot be approximated as linear. Nonlinear EMT has been derived from Hertz-Mindlin interparticle contact mechanics [22, 61] and modified by others 26], offering reasonable albeit not completely satisfactory agreement with experiments [51, 30]. A recently proposed elasticity model encompassing many of the same features as EMT was proposed by Jiang and Liu [42] in 2003. This state-of-the-art formulation has had multiple successes and is well-suited to our end goal of combining with a plasticity model.

\subsection{Effective Medium Theory for bulk granular elasticity}

In the classical work of Hertz, two perfectly elastic spheres that are pressed into contact repel each other with a contact force that depends on the radii of the spheres and the "apparent overlap". For two spheres of identical radius $R$ located at $\mathbf{x}_{1}$ and $\mathbf{x}_{2}$, the normal force contact law is

$$
F_{n}(\delta)=\frac{2}{3} k_{n} R^{1 / 2} \delta^{3 / 2}
$$

where $\delta$ is the normal overlap $(1 / 2)\left(2 R-\left\|\mathbf{x}_{2}-\mathbf{x}_{1}\right\|\right)$. The parameter $k_{n}$ is an effective stiffness equal to $4 G_{g} /\left(1-\nu_{g}\right)$ where $G_{g}$ and $\nu_{g}$ are the shear modulus and Poisson ration of the sphere material.

Presume a granular collection with an isotropic distribution of contacts that undergoes affine deformation when stressed. The Hertzian contact law generalizes to an EMT that gives the following bulk modulus under pure compression:

$$
\kappa=\frac{1}{18 \pi} \Phi Z k_{n}\left(-\frac{1}{3} \operatorname{tr} \mathbf{E}\right)^{1 / 2}
$$

where $\Phi$ is the volume fraction, $Z$ is the mean coordination number, and $\mathbf{E}$ is the (infinitesimal) strain tensor. The nonlinearity can be seen as arising directly from the nonlinearity of Hertz's contact law. The major point is that the bulk modulus scales with (compressive strain) $)^{1 / 2}$, or equivalently as $p^{1 / 3}$ under isotropic compression. This has been verified directly in large-scale DEM simulations of compressed sphere packings both with and without interparticle contact friction [58].

Supposing frictionless spheres, a mean-field shear modulus can also be derived in a similar fashion by analyzing an arbitrary affine deformation including shear strain. One ultimately finds that the bulk and shear moduli under non-zero shear scale similarly to the above form:

$$
\kappa \propto G \propto \Phi Z(-\operatorname{tr} \mathbf{E})^{1 / 2} \propto(\Phi Z)^{2 / 3} p^{1 / 3}
$$

The inclusion of shear strain renders EMT less accurate. The moduli agree with the above scalings but only under low pressures $(<10 \mathrm{MPa})$ [58]. Experiments verify that the shear dependence on $p$ is characterized by a larger exponent at higher pressures [30]. This could be because shearing under higher pressures tends to render the affine displacement assumption less valid. Time-dependent relaxation occurs, which significantly complicates a determination of the shear modulus. Attempts 
to improve the EMT elastic moduli during shear are not vastly affected by including tangential forces in the analysis; the inclusion of Mindlin's tangential force law 61 between spheres does not change the above scalings.

\subsection{The Jiang-Liu granular elasticity law}

In 2003, Jiang and Liu proposed a modified approach to granular elasticity [42]. Rather than continue laboring on a microscopically derivable mean field theory, their aim was to augment the more successful results of EMT with presumptive forms that capture known macroscopic behavior.

They consider an elastic free energy density of the following form:

$$
\psi(\mathbf{E})=B \sqrt{\Delta}\left(\frac{2}{5} \Delta^{2}+\gamma^{2} / \xi\right)
$$

where $B$ is a relative stiffness that can vary with packing fraction. The compressive strain and shear strain are measured respectively by

$$
\Delta=-\operatorname{tr} \mathbf{E}, \quad \gamma=\left|\mathbf{E}_{0}\right|
$$

where for any tensor $\mathbf{A}$, we adopt the notation $\mathbf{A}_{0} \equiv \mathbf{A}-(1 / 3) \operatorname{tr} \mathbf{A}$ and $|\mathbf{A}| \equiv \sqrt{\sum_{i, j} A_{i j}^{2}}$. The dimensionless parameter $\xi$ will be discussed shortly. In the small displacement limit this gives the Cauchy stress

$$
\mathbf{T}=\frac{\partial \psi}{\partial \mathbf{E}}=2 \underbrace{\frac{B \sqrt{\Delta}}{\xi}}_{G(\Delta)} \mathbf{E}_{0}+\underbrace{B \sqrt{\Delta}\left(1+\frac{\gamma^{2}}{2 \xi \Delta^{2}}\right)}_{\kappa(\Delta, \gamma)}(\operatorname{tr} \mathbf{E}) \mathbf{1} .
$$

Under isotropic compression, the pressure is proportional to $\Delta^{3 / 2}$, in agreement with the most successful result of EMT. The shear modulus scales with $\Delta^{1 / 2}$ as in EMT, but the added nonlinearity in the full form of the bulk modulus allows for some important properties not well-represented by EMT.

In an arbitrary state of strain $(\gamma, \Delta)$, the corresponding Drucker-Prager friction state is

$$
\mu=\frac{\tau_{e q}}{p}=\sqrt{2}\left((\gamma / \Delta)^{-1} \xi+(\gamma / \Delta) 0.5\right)^{-1}
$$

By varying the input $r \equiv \gamma / \Delta$, one can show using basic calculus that $\mu$ has a global maximum value of $1 / \sqrt{\xi}$. Thus, by selecting $\xi$ accordingly, we can prevent certain states of friction from ever arising. This is one of the defining features of Jiang-Liu elasticity.

In the work of Jiang and Liu, static materials were the primary concern. Hence, a static yield criterion $\mu_{s}$ was declared and $\xi$ was set to $\mu_{s}^{-2}$ so as to require that no purely elastic state can exist above $\mu_{s}$. The work herein shall attempt to integrate elasticity within a complete elasto-plastic framework, so the basis for selecting $\xi$ will be slightly different. At the point where the stress state reaches $\mu_{\max }$, the elasticity relation loses convexity. The particular condition for convexity is $\left|\mathbf{E}_{0}\right| \leq(p / 2 B)^{2 / 3} \sqrt{2 \xi}$. Figure 1 elucidates the fashion by which $\mu$ increases to its maximum under increasing shear strain. Plots are discontinued in non-convex regions, where the elasticity law is deemed void.

More than just a model that connects with yield, the Jiang-Liu elasticity model has had some convincing successes in representing granular statics:

1. The nonlinear form of $\mathbf{T}$ produces a stiffness tensor $\frac{\partial T_{i j}}{\partial U_{k l}}$ that agrees to a satisfactory extent with the form of the stiffness tensor extracted from experimental data 51].

2. The model predicts Janssen-type saturation of the wall stresses in a tall silo. The ratio of vertical to horizontal compressive stresses in the silo is found to be approximately constant when not close to the walls. This verifies the commonly used notion of a "coefficient of redirection", which has been verified in DEM simulations [6]. 

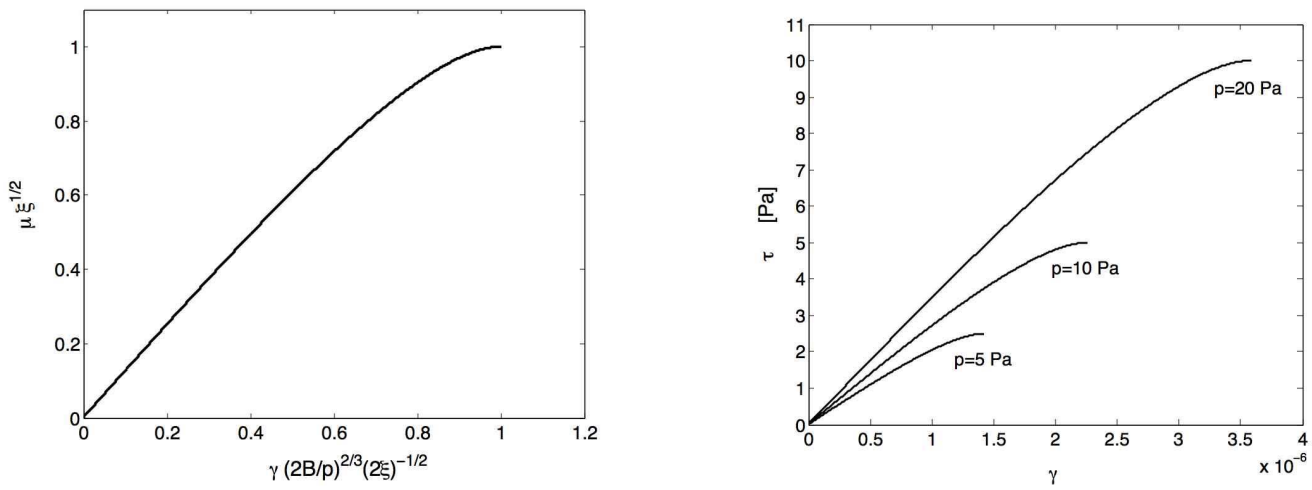

Figure 1: The shear stress vs. shear strain relation under Jiang-Liu elasticity represented as a single dimensionless plot (left), and plotted equivalently as a family of shear stress vs. shear strain curves in SI units using $\xi=4$, $B=7 \times 10^{9} \mathrm{~Pa}$, each curve determined by the applied compressive pressure (right).

3. The model predicts that a granular material under simple shear stress responds anisotropically to the addition of a point-load at a surface. Such anisotropy under pre-stress is a well-known granular phenomenon that is captured appropriately in the nonlinearity of the Jiang-Liu model.

4. It has been observed that preparation history is largely responsible for the stress dip that one often observes under the peak of a sand pile. This fact is reproduced by the elasticity model when solved assuming an initial packing fraction distribution that one might expect for a conical pile constructed from grains flowing out of a nozzle.

\section{Continuum flow}

\subsection{Bagnold scaling and relevant dimensionless quantities}

We now move on to a discussion of granular flow laws for inelastic deformation. Bagnold's seminal work on granular flow followed from shear experiments on granular/fluid suspensions. In the "grain-inertia" regime where the effects of the interstitial fluid are small, Bagnold found that both shear and normal stresses on the shearing wall depend quadratically on the shear rate [6], a phenomenon that came to be known as "Bagnold scaling". Bagnold scaling has been verified for dry grains in a number of experiments and simulations [56, 80, 68, 71, 17]. An explicit form in the case of simple shear of a dense configuration of dry grains can be expressed as the following pair of dimensionless relations:

$$
\begin{aligned}
& \Phi=f(I) \text { for } I=\frac{\dot{\gamma} d}{\sqrt{P / \rho_{s}}} \\
& \mu=g(I) \text { for } \mu=\frac{\tau}{P}
\end{aligned}
$$

In the above, $\Phi$ is the packing fraction, $P$ is the pressure on the shearing plate, $\tau$ is the shear stress, and the steady shear rate is $\dot{\gamma}$. The dimensionless number $I$ is commonly referred to as the inertial number or normalized flow rate, and $\mu$ is the effective friction.

The simplest way to understand these equations is through dimensional analysis. The major physical quantities involved in a gravity-free simple shearing between long rough plates are the material parameters $d$ and $\rho_{s}$, and the variable quantities $\Phi, \tau, P$, and $\dot{\gamma}$. This tacitly ignores the possibility of any other length-scales playing a role and presumes that collisions are fully dissipative 

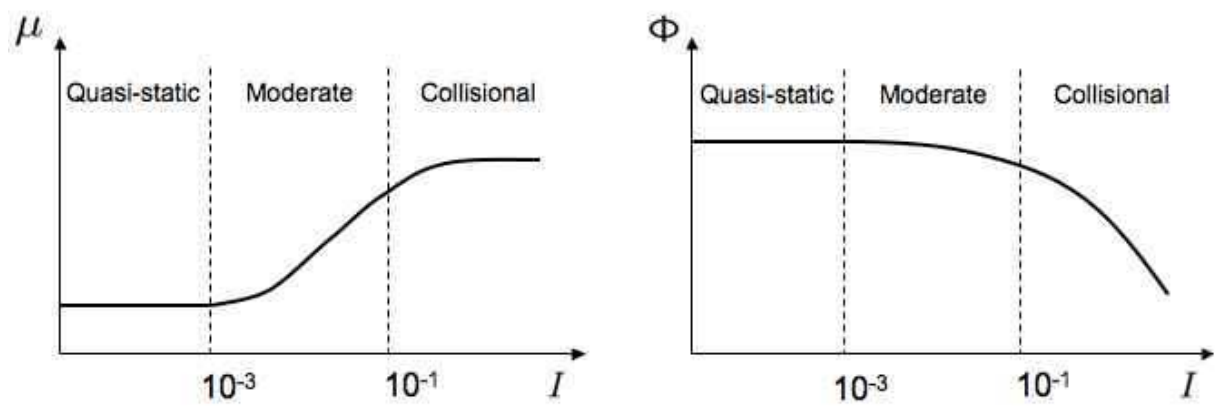

Figure 2: Qualitative diagrams (primarily due to [17]) showing the variation of dimensionless parameters through the various flow regimes under simple shearing. In other geometries, the quasi-static regime of $\mu$ is not as clear to define in terms of $I$ due to meso-scale effects. The coefficient of restitution affects $\Phi$ vs. $I$ in the collisional regime. The moderate regime is relatively well-determined as a sole function of $I$.

(pressure high enough to damp out restitution), two assumptions whose consequences are important and will be discussed shortly. The particle-on-particle contact friction $\mu_{p}$ is also ignored. Granted, $\mu_{p}$ does affect $\mu$, but it has been found to merely translate the $\mu$ vs. $I$ relationship vertically [17].

With these assumptions, the packing fraction and effective friction in a simple shear apparatus should arise uniquely from $P$ and $\dot{\gamma}$ as a matter of cause and effect. Nondimensionalization implies that $\mu$ and $\Phi$ should depend only on $I$. Unlike a Newtonian fluid where a temperature time-scale exists, the quadratic dependence of stress on shear rate can be seen to arise from the fact that $\dot{\gamma}$ can only be scaled by the square root of a stress quantity.

\subsection{Flow regimes}

It is important to clarify the properties and overall validity of equations 5 and 6 over the range of possible flow behaviors. First, zero collisional restitution was presumed in justifying these equations. The assumption is valid if the kinetic energy of a collision is always dissipated in full on impact, presumably in the form of heat and sound.

For faster shear rates, this source of energy loss causes notable rate-sensitivity. Resultantly, $I$ becomes one-to-one with $\mu$, and $\dot{\gamma}$ is immediately determined by $\tau$ and $P$ as in a non-Newtonian fluid. Gathering these properties into a general classification scheme, a flow rate is deemed to be moderate when $I$ is large enough for rate-dependence, but small enough for the flow to stay dense as per the collisional collapse argument. Data of da Cruz et al. [17], would suggest this regime lies within the band $10^{-3}<I<10^{-1}$. In day-to-day terms, the flowing region of an hourglass is typically in the moderate range.

Moderate flows have the property of shearing dilation, where increasing the normalized flow rate causes the steady-state packing fraction to decrease (i.e. $f$ in equation 5 becomes a decreasing function). This should not be confused with shear dilation, which refers to the drop in packing fraction as a function of total shear that occurs to a dense assembly at the beginning stages of a shear deformation. Flows too slow to be deemed moderate may still undergo shear dilation due to packing geometry, but rate effects like shearing dilation only set in for faster flows.

Beyond the moderate range, dilute or collisional flows occur in general for $I>10^{-1}$ and correspond to the breakdown of the zero restitution assumption. When $I$ becomes this large, particle collisions are accompanied by some additional "bounce-back" akin to a gas. The collisions are chiefly binary, and particles rarely maintain long lasting contacts. These flows may require a temperature quantity to store fluctuational energy and can be described with dissipative Boltzmann kinetics. A sand blast could be considered a basic example of the collisional regime. 
On the other side of the spectrum, where $I<10^{-3}$, we enter the quasi-static regime. The packing fraction in simple shear does not vary noticeably with $I$ in the (time-averaged) steady limitthe inertial time is always small enough for the particles to find tight compaction. Without a significant contribution from collisional dissipation, rate-dependence subsides, and more complicated dissipation mechanisms dominate such as frictional contact sliding. The stress/strain-rate relationship becomes singular; driving the system with a range of quasi-static normalized shear rates all give the same time-average value for $\mu$. Soil creep is typically in the quasi-static range.

The discussion thus far has focused on simple shear, where stresses and time-average flow are spatially uniform. While the other regimes display a strong local rheology, quasi-static flows are sensitive to gradients in the fields as expressed through some non-local term with an associated length-scale [59, 8, 47, 4]. For example, consider steady flow in an annular Couette cell. Slow moving material far from the inner wall is observed to constantly creep [59], even though the stress state should be below yield by all common local measures. Indeed, the motion appears to be caused by a non-local effect where faster flow near the inner wall has effectively "bled out" into neighboring material. Grain-level specifics such as roughness, grain shape, and configurational statistics (including wall effects) should affect the non-local flow behavior through the new lengthscale. The size, dynamics, and general interpretation of the length-scale are object of debate, though most agree its size should be on the order of several particle widths.

Observe figure 2 for a schematic view of how $\mu$ and $\Phi$ vary throughout the flow regimes (in simple shear). The major points of this discussion are: moderate flow is much simpler than quasi-static, and collisional flow is outside our interest as it is not dense. Moderate flows are characterized by a local rheology relating $I$ to both $\mu$ and $\Phi$. The regime is definitively rate-dependent and equation 6 inverts into a fluid-like law wherein the flow rate can be determined uniquely from the stress state. As $I$ decreases to the quasi-static limit, however, quantities that were previously ignorable become important. Grain motion is locally correlated at some length-scale causing nonlocality and a larger role of grain-level properties. The flow does not permit a fluid-like treatment as before, since the dissipation is largely rate-independent.

\subsection{Quasi-static flow models}

To review dense flow models, we begin with those aimed at quasi-static behavior. Since the stresses are not a direct function of the flow rate, they are typically obtained by asserting an elasticity law upon a small component of the total deformation. Linear elasticity is often presumed, but this may be an oversimplification that carries more consequences at lower stresses as described in section 3. Critical State Theory [79], Rudnicki-Rice-type modeling [73], the Anand-Gu model [1], and the model of La Ragione et al. [72] each can be used for granular deformation in this fashion. Other models couple to the IFE stress formulation in 2D, such as rate-independent coaxiality [63] and the Stochastic Flow Rule [47].

Some quasi-static theories account for nonlocality, though none have been proven to do so reliably for steady flow in arbitrary 3D environments. Several theories are based on new definitions of temperature, which introduce a length-scale via a "heat equation". These include Shear Transformation Zone (STZ) Theory [54, 27], which relies on an effective temperature governing STZ creation, the dense flow theory of S. B. Savage 78], which defines a granular temperature to measure strain-rate fluctuations, and Edwards statistics [23], which utilizes a temperature-like 'compactivity' derived from an entropy per free volume. These models provide interesting physical insight, but do not appear to be at the point of development that simulating arbitrary flows would be possible - some are restricted to 2D, the boundary conditions for the new temperature are rarely obvious, and the equations may not be closed except in a few symmetric test cases. Besides temperature approaches, nonlocal behavior could also be described with a diffusing order parameter, as in [47, 4], or through a more general strain-gradient plasticity theory [33, 34, 88, 38, 37].

The quasi-static flow regime, though important, appears at the moment too difficult to account for appropriately within a simple 3D continuum framework. Likewise, the following concession 

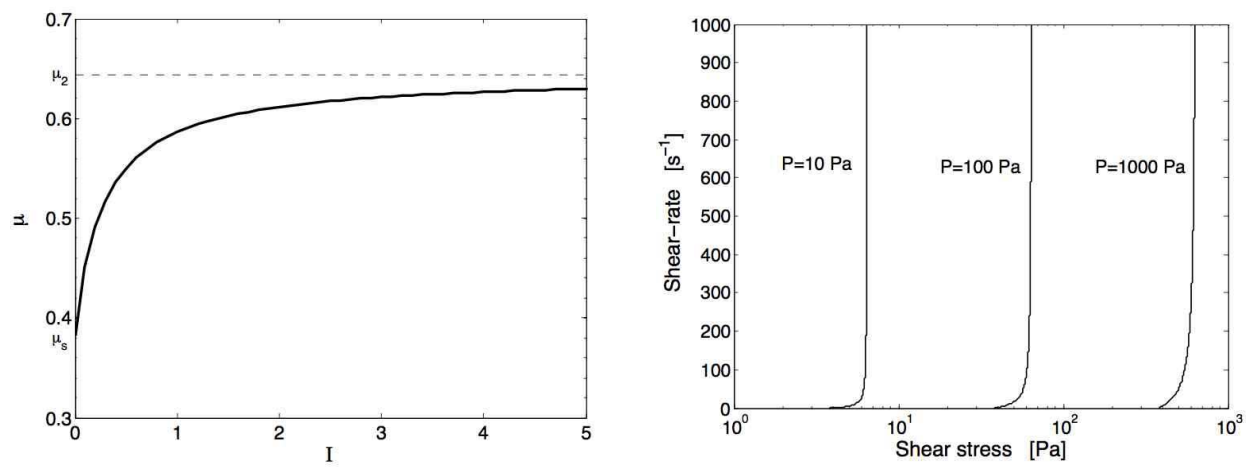

Figure 3: The plastic flow rheology in simple shear plotted as one dimensionless relationship (left), and plotted equivalently as a family of shear-rate vs. shear stress curves in SI units, each curve determined by the applied compressive pressure (right).

is enacted: The model to be constructed in this work shall neglect quasi-static flow behavior altogether, opting instead to combine a static response directly with moderate flow rheology. The model herein may ultimately serve as the backbone for a fuller model that also incorporates the dependence of a length-scale on the slow dynamics. This possibility shall be considered in more depth when we compare predictions of the model directly to experimental/DEM data. But for now, we accept inaccuracy in describing quasi-static motion in exchange for a closed, general model capable of giving worthwhile predictions over the full range of dense material behavior, accounting for both statics and flow.

\subsection{Moderate flow law of Jop et al.}

A closed form law to predict moderate flow must now be discussed. Since the moderate flow regime is (monotonically) rate-dependent, the function $g$ in equation 6 should be invertible. Hence, increasing the normalized shear rate $I$ results in higher $\mu$. This notion may seem counterintuitive to the fundamental idea that $\mu$ should decrease to a kinetic value as the rate of sliding picks up. But recall, for moderate flow rates, the impact dissipation dominates sliding affects. In slower or transient flows, shear weakening is indeed observed for "overconsolidated" material, and accounted for in various models via hardening parameters [1, 79].

Based on results from numerical simulations of planar shear [17, 40], and successful extensions to plane-strain inclined chute flows [59, 80], the experiments of [43] were conducted to quantify $g^{-1}$ for glass beads:

$$
I=g^{-1}(\mu)=I_{0} \frac{\mu-\mu_{s}}{\mu_{2}-\mu} \text { for } \mu>\mu_{s} .
$$

The values of the parameters were measured at $I_{0}=0.279, \mu_{s}=\tan 20.9^{\circ}$, and $\mu_{2}=\tan 32.76^{\circ}$. The relation states that the normalized shear rate $I$ increases as the material is sheared with higher $\mu$. But $\mu$ must exceed some static yield value $\mu_{s}$ before any plastic flow ensues. There is also some maximal $\mu$ value, $\mu_{2}$, and all steady shear flowes should be tenable for a value of $\mu$ less than $\mu_{2}$. Consequently, if applied stresses exceed $\mu_{2}$ the flow is deemed accelerative with no apparent steady state. While this last point may be somewhat of an oversimplification from a physical point of view, the approximation is helpful especially in light of the fact that $\mu$ for dense flow is usually well below $\mu_{2}$.

The first attempt to extend equation 7 into 3D was met with high success. In Jop et al. [44], codirectionality was applied, presuming that the deformation rate tensor

$$
\mathbf{D}=(1 / 2)\left(\boldsymbol{\nabla} \mathbf{v}+(\boldsymbol{\nabla} \mathbf{v})^{T}\right)
$$


is proportional to the deviatoric stress tensor

$$
\mathbf{T}_{0}=\mathbf{T}-(1 / 3)(\operatorname{tr} \mathbf{T}) \mathbf{1} .
$$

Written in full, Jop proposed the following generalization of equation 7

$$
\mathbf{D} \frac{d}{\sqrt{P / \rho_{s}}}=I_{0} \frac{\mu-\mu_{s}}{\mu_{2}-\mu} \frac{\mathbf{T}_{0}}{\tau}
$$

where now $P=-(1 / 3) \operatorname{tr} \mathbf{T}$ and $\mu=\tau / P$ where $\tau=\left|\mathbf{T}_{0}\right| / \sqrt{2}$ is the equivalent shear stress. When $\mu<\mu_{s}$, we take $\mathbf{D}=\mathbf{0}$ establishing a Drucker-Prager yield criterion. Since the flow condition being used is codirectionality, the flow rule is thus non-associative.

Since $\mathbf{D}$ is proportional to a deviatoric tensor, the flow rule asserts plastic incompressibility. While, as previously noted, dilation in dense flow does occur, it is typically on the order of only a few percent and quickly reaches a steady value over large deformations. Moreover, $\Phi$ is unnecessary to compute the steady shear rate, hence the approximation of plastic incompressibility should have negligible effect on the velocity field of a dense steady flow. Note that evolution laws for the packing fraction have not yet been quantified in this context. Some quasi-static flow models attempt this $[1,79,73]$ but rather than try to modify one of these, we go along with the presumptions of Jop et al. and ignore plastic dilation. The bigger impact of this assertion is not on the flow, but rather on the stresses in the static regions - If plastic flow transiently passes through a region that is ultimately static in the steady-state, the dilation that occurred there affects the local elastic moduli.

The flow model was tested experimentally in a chute apparatus with rough sidewalls and bottom to induce non-trivial 3D flow. Results were shown to match experiments to a high degree (always within $15 \%$ ) even while varying several parameters (e.g. inclination angle, flow height and width). With positive experimental validation, the flow model became one of the first to describe welldeveloped, inhomogeneous, 3D, dense granular flows under moderate flow rates.

We now proceed to unite the Jop et al. plasticity law (equation 8) with the Jiang-Liu elasticity law (equation 4).

\section{Combining elasticity and plasticity}

\subsection{Kinematics}

First, some kinematic quantities of interest to the discussion shall be briefly reviewed (see [35] or [2] for more details). For a material element that begins at location $\mathbf{X}$ and resides at $\mathbf{x}$ some time $t$ later, the motion function $\chi$ is defined by $\mathbf{x}=\chi(\mathbf{X}, t)$. The elastic and plastic responses are combined using the Kröner-Lee decomposition of the deformation gradient $\mathbf{F} \equiv \partial \boldsymbol{\chi}(\mathbf{X}, t) / \partial \mathbf{X}$ [50, 52]:

$$
\mathbf{F}=\mathbf{F}^{e} \mathbf{F}^{p}
$$

The decomposition models the deformation of an element at any time as a progression of two stages of deformation. First, the unstressed reference configuration is deformed by the plastic deformation $\mathbf{F}^{p}$ to an intermediate configuration, which is still deemed as stress-free. From there, the material deforms elastically via $\mathbf{F}^{e}$ to its final or deformed configuration. The intermediate configuration can be thought of as the residual state that occurs when all stresses on a deformed volume element are released, thereby unloading all elastic mechanisms, leaving only the total plastic deformation.

The spatial velocity gradient can be expressed in terms of $\mathbf{F}$ via

$$
\mathbf{L} \equiv \frac{\partial \mathbf{v}}{\partial \mathbf{x}}=\dot{\mathbf{F}} \mathbf{F}^{-1}
$$


where always represents the material time derivative. Generalizing this, the elastic and plastic velocity gradients are defined as

$$
\begin{aligned}
\mathbf{L}^{e} & =\dot{\mathbf{F}}^{e} \mathbf{F}^{e-1} \\
\mathbf{L}^{p} & =\dot{\mathbf{F}}^{p} \mathbf{F}^{p-1}
\end{aligned}
$$

which in turn enables definitions of the elastic and plastic deformation rate and the elastic and plastic spin:

$$
\begin{aligned}
\mathbf{D}^{e} & =\operatorname{sym}\left(\mathbf{L}^{e}\right) & & \mathbf{D}^{p}=\operatorname{sym}\left(\mathbf{L}^{p}\right) \\
\mathbf{W}^{e} & =\operatorname{skw}\left(\mathbf{L}^{e}\right) & & \mathbf{W}^{p}=\operatorname{skw}\left(\mathbf{L}^{p}\right) .
\end{aligned}
$$

The deformation gradient and its elastic/plastic counterparts are presumed to have positive determinant, permitting the polar decomposition

$$
\mathbf{F}^{e}=\mathbf{R}^{e} \mathbf{U}^{e}
$$

where $\mathbf{U}^{e}$ is the elastic right stretch (which is symmetric positive definite) and $\mathbf{R}^{e}$ is the elastic rotation. The plasticity model we wish to use is incompressible and as such we have $\operatorname{det} \mathbf{F}^{p}=1$. Consequently, $\operatorname{det} \mathbf{F}^{e}=\operatorname{det} \mathbf{F}$. From the elastic stretch, we invoke the material Hencky strain measure to define the elastic strain:

$$
\mathbf{E}^{e}=\log \left(\mathbf{U}^{e}\right)
$$

\subsection{Elasto-plastic constitutive picture}

The constitutive framework to be used herein is analagous to a spring/damper combination in series, with Jiang-Liu elasticity representing the spring deformation and the flow law of Jop et al. representing the damper (with a yield criterion). Notably, the stresses are modeled as being determinable everywhere from the field $\mathbf{E}^{e}$. When the resulting stresses satisfy yield, the plastic flow law determines the flow-rate $\mathbf{L}^{p}$.

It is a non-trivial physical assertion to model all stresses in a dense flowing granular material as being derivable from the elastic deformation of the grains. Other microscopic stress agencies exist such as internal viscous damping and fluctuational stresses. To validate our assertion, discrete particle simulations of Rycroft 74] were performed in several dense, inhomogeneous flow environments. The instantaneous stress tensor over an element is represented by the particulate formula

$$
\mathbf{T}=\frac{1}{V}\left(\sum_{i<j}^{N} \mathbf{r}^{(i j)} \otimes \mathbf{f}^{(i j)}-\sum_{i=1}^{N} m^{(i)}\left(\mathbf{v}^{(i)}-\overline{\mathbf{v}}\right) \otimes\left(\mathbf{v}^{(i)}-\overline{\mathbf{v}}\right)\right)
$$

for $N$ the number of grains in the element, $V$ the element volume, $\mathbf{f}^{(i j)}$ the contact force of particle $i$ on particle $j, \mathbf{r}^{(i j)}$ the vector connecting the centroids of particles $i$ and $j, m^{(i)}$ the mass of particle $i$, $\mathbf{v}^{(i)}$ the velocity of particle $i$, and $\overline{\mathbf{v}}$ the average velocity over all $N$ particles. The first term inside the parenthesis represents a solid-like stress response derived from contact forces, while the latter term is gas-like, accounting for stresses due to velocity fluctuations. Rycroft found that the gas-like term is always exceedingly small compared to the solid term. The contact law used in the simulation was visco-elastic (plus sliding friction) giving the force decomposition $\mathbf{f}^{(i j)}=\mathbf{f}^{\mathbf{e}^{(i j)}}+\mathbf{f}^{\mathbf{v}(i j)}$. By comparing the viscous and elastic force contributions, Rycroft found that the elastic contribution is always vastly dominant. Altogether,

$$
\mathbf{T} \cong \frac{1}{V}\left(\sum_{i<j}^{N} \mathbf{r}^{(i j)} \otimes \mathbf{f}^{\mathbf{e}(i j)}\right) .
$$

This evidence suggests the elastic mechanism accounts for virtually all stress in a flowing or static element of dense granular material, lending reasonable validation to our proposed elasto-plastic treatment. 


\subsection{Modifying the Jiang-Liu elasticity law}

The Jiang-Liu elasticity law requires some slight modifications in order to be used for elastoplasticity. Since flowing materials can have $\mu$ above $\mu_{s}$, the elastic law must permit these stress states. Hence, we propose the following important modification to Jiang-Liu elasticity: Set $\xi=\mu_{2}^{-2}$ instead of $\xi=\mu_{s}^{-2}$. Little loss in representing elasticity occurs from this change. The $\xi$ parameter was engendered to represent the macroscopic repose angle of a static granular assembly; it was not determined from any quantitative microscopic requirements.

With $\xi=\mu_{2}^{-2}$, there can never be an elastic stress state that has $\mu>\mu_{2}$. Looking back to equation 8 , notice that the flow rule cannot apply to a stress state that exceeds $\mu_{2}$. Since the JiangLiu law admits a cap on the value of $\mu$, this property prevents elastic stress states from entering the forbidden regime of the Jop et al. flow law, which offers several benefits when attempting to numerically solve for flow. Analytically speaking, capping the elastic stresses at $\mu_{2}$ is not necessary for a solution; a cap above $\mu_{2}$ would also be acceptable, though the plastic response would always preclude such states from arising. Regardless, $\mu_{2}$ is a natural choice that minimizes the extent of alteration of Jiang and Liu's original formulation.

\subsection{Mathematical specifics}

With the motivation provided, we now go about providing a mathematically rigorous framework to unite the proposed elastic and plastic responses. The following is based on a form for thermodynamically compatible elasto-plasticity developed in [2]. An abbreviated discussion shall be provided here. More details can be found in [46].

At the outset, Newton's equations of motion must be upheld for force and torque balance, and mass is conserved:

$$
\frac{\partial}{\partial \mathbf{x}} \cdot \mathbf{T}+\rho \mathbf{g}=\rho \dot{\mathbf{v}}, \quad \mathbf{T}=\mathbf{T}^{T}, \quad \rho=\rho_{0}(\operatorname{det} \mathbf{F})^{-1}
$$

where $\rho_{0}$ is the initial material density, equal to random-close-packing $(63 \%)$ times $\rho_{s}$. To institute simultaneous elastic and plastic constitutive responses, three major restrictions are enforced

1. Frame-indifference

2. Non-violation of the second law of thermodynamics

3. Isotropy of the reference and intermediate configurations.

The presumption of isotropy, particularly in the intermediate state, deserves some attention. Anisotropy of plastically deformed granular material has been studied extensively both in 2D [84] and in 3D where it has been quantified [87] and modeled [90, 86] often by inclusion of a fabric tensor. These studies show varying levels of anisotropy that evolve over a range of strains that are large, but always significantly less then $50 \%$. In the limit of well-developed flows, however, experimental evidence of [85] and discrete simulations of [19, 76] support the use of an isotropic flow law.

With the presumed $\mathbf{F}^{e} \mathbf{F}^{p}$ decomposition, one can show via power conjugacy that the stress interacts with the elastic and plastic mechanisms through a surrogate known as the Mandel stress

$$
\mathbf{M}=J^{e} \mathbf{F}^{e T} \mathbf{T} \mathbf{F}^{e} .
$$

To connect to more common stress measures, $\mathbf{M}$ is equivalent to $\mathbf{C}^{e} \mathbf{T}_{\mathrm{II}}$ where $\mathbf{T}_{\mathrm{II}}=J^{e} \mathbf{F}^{e-1} \mathbf{T} \mathbf{F}^{e-T}$ is the second Piola stress as measured using $\mathbf{F}^{e}$ instead of $\mathbf{F}$, and $\mathbf{C}^{e}=\mathbf{F}^{e T} \mathbf{F}^{e}$ is the elastic right Cauchy-Green tensor. As in the analogy with a spring and damper in series, the Mandel stress determines both the elastic strain and the rate of plastic flow. Equations 4 and 8 are written in this parlance to give the elasto-plastic constitutive relations:

Elasticity relation:

$$
\mathbf{M}=2 G \mathbf{E}_{\mathbf{0}}^{\mathbf{e}}+\kappa\left(\operatorname{tr} \mathbf{E}^{\mathbf{e}}\right) \mathbf{1}
$$


where $\kappa=B \sqrt{\Delta}\left[1+\gamma^{2} /\left(2 \Delta^{2} \xi\right)\right]$ and $G=\sqrt{\Delta} B / \xi$, for $\Delta=-\operatorname{tr} \mathbf{E}^{e}$ and $\gamma=\sqrt{\mathbf{E}_{0}^{e}: \mathbf{E}_{0}^{e}}$. If $\operatorname{tr} \mathbf{E}^{\mathbf{e}}>0$, both $\kappa$ and $G$ are 0 .

Plastic flow rule:

$$
\mathbf{L}^{p}=\mathbf{D}^{p}=\frac{I_{0}}{d} \sqrt{\frac{P}{\rho_{s}}} \frac{\mu-\mu_{s}}{\mu_{2}-\mu} \frac{\mathbf{M}_{0}}{\tau}
$$

where $P=-\operatorname{tr}(\mathbf{M}) / 3, \tau=\sqrt{\mathbf{M}_{0}: \mathbf{M}_{0} / 2}$, and $\mu=\tau / P$. If $\mu<\mu_{s}$, then $\mathbf{D}^{p}=\mathbf{0}$.

As for the initial conditions, we declare that the granular body begins free of any plastic deformation, and that the body's initial deformation increment is elastic:

$$
\mathbf{F}^{p}(t=0)=\mathbf{1}, \quad \mathbf{L}^{p}(t=0)=\mathbf{0} .
$$

The granular model being proposed in this paper is fully defined by the closed system of equations 9, 11, 12, 13, 15, 16, 17, and 18 along with these initial conditions.

\section{Implementation and results}

The above equations were solved numerically using the ABAQUS/Explicit software package (from ABAQUS 6.5) with the constitutive behavior coded as a Vectorized User Material (VUMAT) model. In line with our interests, we choose three standard geometries that eventually have a sustained, well-developed flow response:

- Long inclined chute

- Annular Couette cell (with downward gravity)

- Wide flat-bottom silo flow

The simulations are run long enough for transient behavior to vanish. We may refer to this as "steady" flow, but a more general meaning is implied, since environments like silo drainage do not correspond to an Eulerian boundary value problem (the top surface always descends). Beyond visual observation, the disappearance of transients is quantifiable in the total kinetic energy, which becomes constant (compared to the kinetic energy transients) when flow is well-developed.

\subsection{Numerical considerations: The method of inertial density reduction}

ABAQUS 6.5 implements Lagrangian finite element deformation, presenting some challenges in the modeling of steady flow behavior. In particular, there are two major numerical concerns that shall now be addressed:

1. The steady response generally emerges after a high magnitude of deformation. However, accuracy decreases dramatically when element distortion is too large. Arbitrary Lagrange-Eulerian (ALE) routines can be used to counteract this by periodically sweeping the mesh to a less distorted configuration. However, we find that the convection of variables between mesh sweeps carries unacceptably large error especially near boundaries, where spatial gradients have a reduced sample space. Later versions of ABAQUS define purely Eulerian elements, which may improve this situation. However, as will be discussed momentarily, a different remedy can be instituted that uses the typical formulation. 
2. The constitutive relations are increasingly sensitive in the low pressure regime. By equation 18. low pressure material at the onset of yield plastically flows at a rate proportional to $P^{-1 / 2}$, which can cause rapid variation in $\mathbf{D}^{p}$ during the transient phase. From an implementation standpoint this induces numerical stiffness; the rate of change may be too large to be adequately represented by the stable time increment. Furthermore, by equation 17, the elastic moduli vanish sharply with decreasing pressure: $\kappa \propto P^{1 / 3}$ as $P \rightarrow 0$. Once again, this requires that the time step be small enough at low pressures so as to accurately track the changes in elastic properties. Numerical stiffness of the type just described is an important consideration since several of the flow environments to be investigated utilize the traction-free boundary condition, inducing non-negligible regions of low pressure in the material body.

A single encomassing remedy, novel to this author's knowledge, was instituted to resolve both these issues: the inertial density of the material was artificially reduced by several orders of magnitude, holding the gravitational density fixed. The true granular material density (roughly 1500 $\mathrm{kg} / \mathrm{m}^{3}$ for glass beads) was used in body force computations in order to accurately represent the force of gravity. However, the density used in computing the inertial force $\rho \dot{\mathbf{v}}$ was artificially decreased by factors on the order of $10^{4}-10^{5}$. In systems without excessive curvature, the inertial force should vanish in steady state, so this alteration does not impact the correctness of the welldeveloped flow solution. To be clear, density reduction is performed only on the unstressed reference body, so that mass conservation is upheld during flow.

By decreasing the inertial density, the wave speed increases, causing transients to pass quickly in physical time. This allows the steady state velocity and stress fields to emerge faster than the time necessary for large distortions to occur to the body (and likewise the mesh). A clear analogy for this effect can be observed in a rudimentary mass/spring/damper system in seriesunder applied force, the distance over which the motion of the mass is unsteady decreases as mass decreases. Using this technique, most simulations of the granular model reached steady state before any nodal displacements were noticeable to the eye. At the same time, since the stable time increment is proportional to $\sqrt{\rho}$, high enough density reduction reduces the time step (relative to the deformation speed) below the threshold necessary to accurately represent low pressure material during the transient phase. Hence, artificial density reduction confronts both issues raised above. It should be noted though, that there is no apparent benefit in terms of computation time when using this method since the transient time period and the stable time increment both decrease. More details on the method can be found in [46].

\subsection{Verifying the numerical model}

Throughout this work, the model's six parameters are assigned the following values:

\begin{tabular}{|l|l|}
\hline$B=7 \times 10^{9} \mathrm{~Pa}$ & $I_{0}=0.279$ \\
\hline$\rho_{s}=2450 \mathrm{~kg} / \mathrm{m}^{3}$ & $d=0.003 \mathrm{~m}$ \\
\hline$\mu_{s}=\tan \left(20.9^{\circ}\right)$ & $\mu_{2}=\tan \left(32.76^{\circ}\right)$ \\
\hline
\end{tabular}

Recall that $\xi$ is also a parameter, but its value is tied directly to the value $\mu_{2}$. Except for $d$, these values were all lifted from Jiang and Liu [42] and Jop et al. 44]. As both groups considered spherical glass beads, it is assumed that their data should be representative of the same material. The particle diameter is set to $3 \mathrm{~mm}$, as this is the common value used in the experiments and DEM simulations of the MIT Dry Fluids Group, whose data this model will be compared against later. It should be noted that the particle diameter easily scales out in the non-dimensionalization. The elements used, unless otherwise stated, are hexahedral of type C3D8R in ABAQUS/Explicit.

To check the implementation of the material model, one element tests were performed wherein a box element is compressed laterally with a fixed pressure, and sheared tangentially with various shear tractions. Implicit and explicit numerical integration routines were encoded into VUMAT files, and it was verified that both give steady flow rates that match the analytical form (equation 
(7) to high accuracy. Since explicit integration is only conditionally stable, the time step must be sufficiently small in order for numerical stability at the higher shear stress values. The implicit routine is always stable numerically, but for higher shear stresses, the time step must be reduced to ensure convergence of the Newton-Raphson solver. For the simulations detailed in this work, the time step is always small enough for explicit stability, a byproduct of the artificial density reduction. Together with the fact that explicit integration is computationally faster, the choice was made in the upcoming simulations to use the explicit routine.

\subsection{Rough-walled inclined chute}

Figure 4(a) reviews the geometry and boundary conditions for the rough inclined chute environment, which was originally studied by Jop et al. . In that work, equation 8 was directly solved as a non-Newtonian Bingham fluid, letting viscosity go to $\infty$ below yield. The approach delivers a steady velocity computation (with some numerical difficulty near the rigid interface), but by avoiding elasticity altogether, no stresses can be computed in the rigid zones. In terms of completeness, the Bingham fluid approach is useful, but not equipped to handle the general traction/kinematic boundary value problem. The elasto-plastic formulation herein is complete but has added many new relationships and a different kinematical perspective on deformation. However, the predicted steady flow should not differ noticeably from the Bingham flow, as elastic stretching vanishes in steady-state. By analyzing this case, we hope to show sufficient agreement between the two solutions, ensuring the elasto-plastic model has inherited the successes of the Bingham flow solution in this environment [4].

The chute is long such that the flow can be deemed invariant in the $z$ direction. The bottom of the chute is at $y=60 d$ and the chute is $142 d$ wide. The angle of incline of the chute with respect to the horizontal is $22.6^{\circ}$.

The chute model uses 2600 box elements arranged in a grid 65 elements wide in the $x$ direction by 40 in the $y$. The assembly is only 1 element wide in the $z$ direction - symmetry conditions were invoked by constraining the nodes on the back face of the $x y$ slab to move with the same displacements as their front face counterparts. The simulation must start in a reference state with no gravity. Gravity is then ramped up to its full value. Since the material must be compressed before any shear stress can be supported, the components of gravity are applied one at a time; the $y$ body force is first smoothly ramped up over $1 \times 10^{-5}$ s to its final value of $f_{g} \cos \left(22.6^{\circ}\right)$, where $f_{g}=0.63 g \rho_{s}$. After a $2 \times 10^{-6}$ s pause for any needed relaxation, the component pointing down the chute is smoothly ramped up to $f_{g} \sin \left(22.6^{\circ}\right)$ over $2 \times 10^{-5}$ s. The assembly is then left to flow until a total time of $5 \times 10^{-4}$ s has elapsed.

The inertial density was artificially decreased by a factor of several hundred thousand to a value of $\rho=2 \times 10^{-3}$. At the free surface, theoretically, the pressure goes to zero causing the elastic moduli to vanish. This is dangerous for procedures such as ABAQUS/Explicit that consistently send waves through the material - any small perturbation could cause a node to accelerate out of control. Moreover, the flow rule becomes undefined. To keep the free surface in tact, a few Pascals of overpressure are applied.

While the flow is eventually very steady to the eye, a more quantitative measure is desired. From $t=0-4 \times 10^{-5} \mathrm{~s}$, the motion is markedly transient due to gravity ramping up. The system then relaxes toward the steady state, first rapidly but then slowly as steady state is approached. Comparing the system's rate of relative kinetic energy change in the fast relaxation period to its value at the end gives a rough criterion for how steady a flow is. At the end of the run, the rate of decrease of the relative kinetic energy is over 500 times smaller than the value it takes during the initial relaxation, which indicates a strong steady-state behavior.

Comparisons to the numerical results of Jop et al. are displayed in figure «(b). The agreement is quite good considering how disparate the solving methods are. Jop utilized a fixed grid finitedifference scheme solving a non-Newtonian Navier-Stokes-type problem, while the elasto-plastic results were obtained by a Lagrangian explicit procedure on nodes that are constantly moving 
(a)

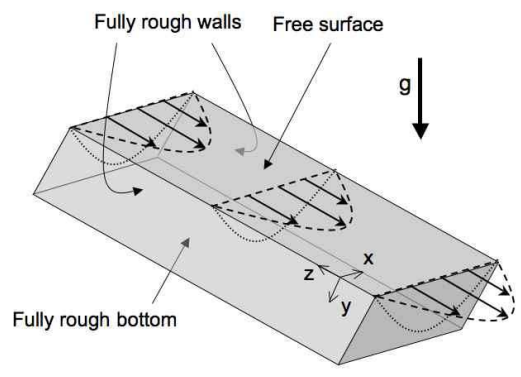

(b)

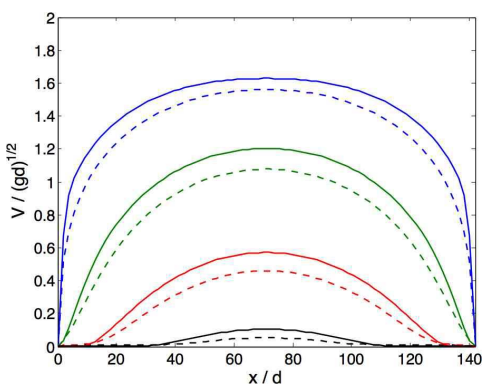

(c)

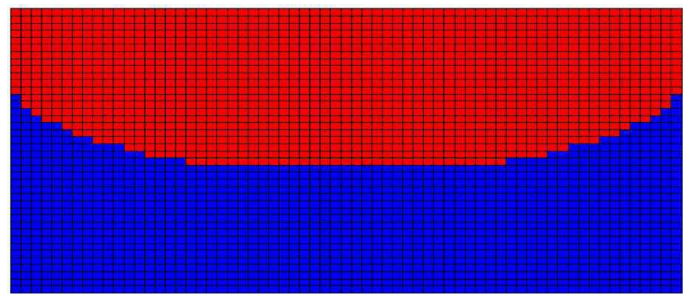

(d)

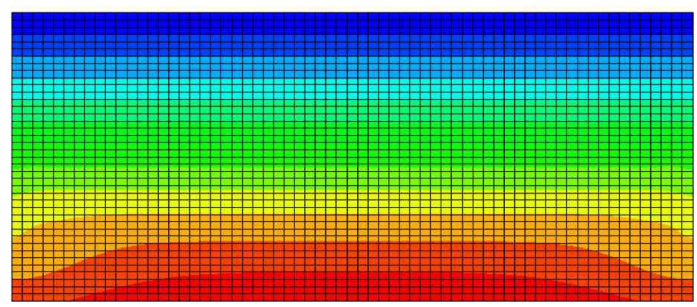

Figure 4: (a) The rough inclined chute setup. (b) The velocity as a function of $x$ at depths $y / d=0,9.2,18.4,27.6$. The dashed curves are the numerical results of Jop et al. [44] via finite differences with a Stokes-type solver. The solid curves are from the steady solution of the elasto-plastic model implemented on ABAQUS/Explicit. (c)-(d) Elasto-plastic results viewing the $x y$ plane with $y$ downward: In (c), regions of plastic flow are in red and static in blue. In (d), the normalized pressure $P / \rho g d$ is displayed.

during steady-state. While differences of numerical procedure can cause different sources of gain and loss, it seems more likely that the discrepancies are stemming from the free surface condition. The true deformation rate at the top corners is actually infinite. As this is numerically impossible for either scheme, large but finite gradients occur there numerically as determined by the choice of the free surface treatment. This has a clear trickle-down effect on the global velocity field and, in lieu of the particular fashion in which the solutions differ, we suspect this effect causes much of the difference between the two numerical data sets.

One distinguishing feature of the elasto-plastic model is its ability to calculate both flow and stress everywhere. Figure 4 (d) displays the pressure distribution over the full geometry. Note that the stresses vary smoothly through the transition from yielding to static, as opposed to the interfacial stress issues that commonly occur with Bingham fluid models. In particular, observe that the pressure field goes from decreasing linearly in the flowing zone, to decreasing somewhat nonlinearly in the static zone. In this geometry, compressive stresses in the $x, y$ and $z$ directions of the steady flowing zone must all be identical under codirectionality. A hydrostatic pressure profile is induced as a result. But upon descending below the flowing layer, codirectionality no longer has this influence on the stresses and a somewhat more complicated elasto-static form for the pressure field becomes apparent. Discrete simulation data for this environment would be helpful for checking the validity of the stress profile in the static zone. Notably, the primitive fully-rough boundary conditions used may not be the most accurate reflection of the true conditions on the bottom and side walls.

\subsection{Annular Couette cell}

We now move on to the annular Couette cell, an environment which has no previously known solution to the Jop et al. flow law. The results shall be compared directly against the myriad of data 
on this environment compiled by G. D. R. Midi [59] and thus the geometric specifics and boundary conditions were chosen so as to give a good representation of the conditions used in these studies. Referring to figure 5(a) for general details on the environment, the following values were selected: $\omega_{\text {wall }}=1.25 \mathrm{rad} / \mathrm{s} \approx 0.2 \mathrm{rev} / \mathrm{s}$, the distance from inner to outer wall is $x_{\text {out }}=30 d$, the height is $z_{\text {bottom }}=10 d$, and the inner wall radius is $40 d$. At the walls, the material motion must match the wall motion in the $\theta$ and $x$ directions, but material can slide without resistance up and down the walls.

Since flow and stress should be symmetric in the $\theta$ direction, the behavior as seen in a downward cut through the annular trough should represent the global behavior. A narrow sector of the annulus (total angle $0.1^{\circ}$ ) was likewise simulated using periodic boundary conditions on the front and back faces - nodal displacements on the front face are constrained to be identical to those on the back face except rotated appropriately by $0.1^{\circ}$. The sector is modeled using 40 elements in the $x$ direction, 15 in the $z$, and a thickness of one element in the $\theta$ direction, for a grand total of 600 elements. Almost all the motion is known to occur near the inner wall in this environment. After preliminary tests of the elasto-plastic model produced the same conclusion, a bias was utilized to crowd nodes closer to the inner wall and improve precision. The bias resulted in half of the elements occurring within a distance of $6 d$ from the inner wall.

The inertial density was reduced by a factor of over one hundred thousand to a value of $\rho=0.01$. First, gravity is smoothly ramped up to its full value over $t=0-1 \times 10^{-5} \mathrm{~s}$. During this time period, as before, a slight overpressure is also applied to the free surface for stability. The rotation of the inner wall then commences, smoothly ramping up to a final value of $\omega_{\text {wall }}=1.25 \mathrm{rad} / \mathrm{s}$ over $t=1-2.5 \times 10^{-5} \mathrm{~s}$. The simulation is then left to flow until a total time of $t=1 \times 5^{-4} \mathrm{~s}$ has elapsed.

Once the inner wall rotation reaches $\omega_{\text {wall }}$, the flow soon after begins to relax toward a macroscopically visible steady-state. Evidencing the fact that the system has indeed reached sufficient steady-state by the end of the run, the rate of kinetic energy loss of the system at the end of the simulation is over three orders smaller than the loss rate immediately after the ramp-up.

Observing figures 5(b) and 5(c), we notice a few major qualitative points. For one, the flow forms a clear shear band near the inner cylinder, a fact corroborated by the provided data. While the predicted thickness is somewhat smaller than found experimentally, the fact that it is on the same order is a major highlight for a model with no added fitting parameters (though we take this point with a grain of caution as shall be discussed momentarily). The authors of [44] expressed their belief that the flow rule would be incapable of describing narrow shear bands. However, the above results show quite clearly that this is not the case for the elasto-plastic model.

Another observation is that the velocity profile does not vary to any observable extent in the $z$ direction. This result has been verified in DEM simulations of this environment [48], where it was found that almost no vertical fluctuation occurs in the fast zone near the inner wall. Of course, the elasto-plastic velocity field dies off much differently than experiment. Where the experimental data is shown to be well-fit with an exponential decay that extends throughout the Couette cell, the elasto-plastic solution predicts a sharp cutoff around $5.5 \mathrm{~d}$ from the inner wall. This result should come as no surprise, since the model does not account for quasi-static, non-local behavior, of which slow exponential decay is a textbook case.

Viewing figure 5(d), it is clear that while the velocity appears invariant in the $z$ direction, other important stress-based quantities are not. The stress ratio $\mu=\tau / P$ indeed has a highly non-trivial spatial distribution, reflective of the nonlinear dependence of the stress state on the plastic flow.

Experimental data on this flow environment indicate that the shear band width stays relatively constant for a range of slower wall speeds [59], and grows with increasing wall velocity in the faster flow regime [49]. As expected from rate-sensitivity, we have verified with multiple simulations that the elasto-plastic model agrees with the latter case: the shear band width correlates positively with inner wall speed (holding gravity constant). We expect this correlation to falsely persist even as the wall speed becomes small. This point serves as a careful reminder that the model has no internal length-scales and requires user discretion when assessing flow predictions - one must check that the 


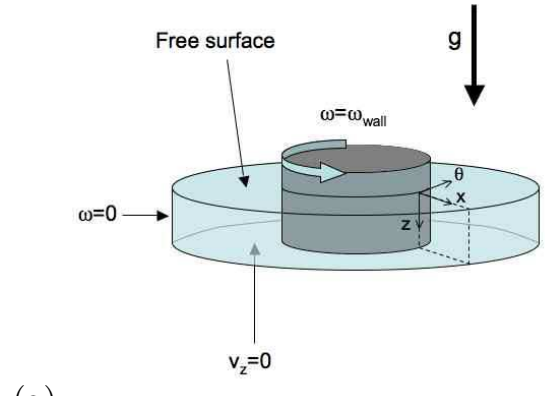

(a)

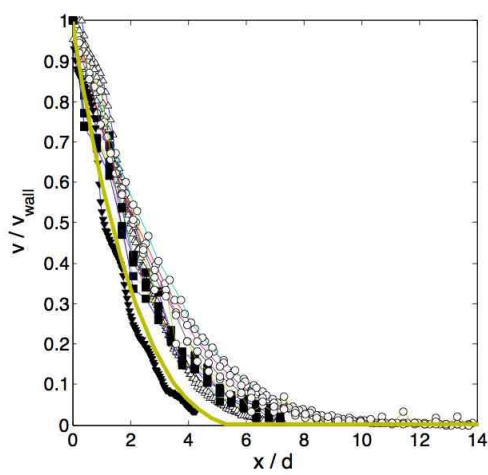

(b)
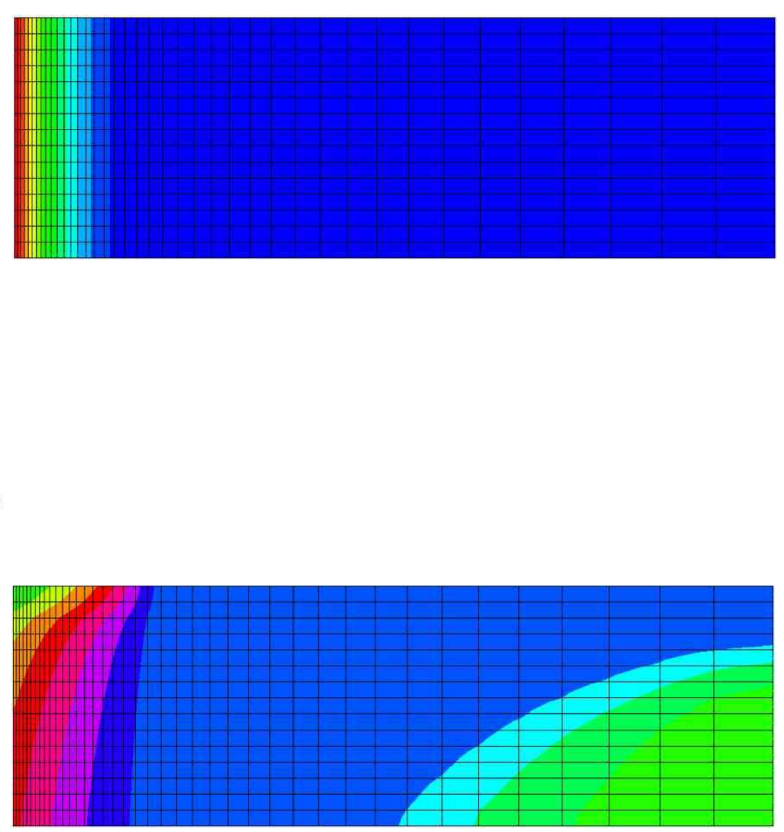

Figure 5: (a) The annular Couette setup. (b) The velocity profile normalized by the wall speed as predicted by the elasto-plastic model at half-height (thick green line) compared against fifteen experimental and discrete element simulation data sets for this type of flow as compiled in [59]. In (c)-(d) the $x z$-plane is shown with $z$ downward: (c) The velocity normalized by the wall speed. (d) The stress ratio $\mu$.

normalized flow rate and shear-rate gradients obey the limitations outlined in sections 4.2 and 2

\subsection{Flat-bottomed silo}

While the past two environments have focused on comparing velocity profiles, the stresses have yet to be directly tested. Experimentally, the stress tensor is a difficult quantity to measure. The stresses in 2D disk assemblies can be approximated using photoelastic grain material, however, there is not currently an experimental method available to measure the stress tensor within an arbitrarily flowing 3D granular material. For this measurement, the best option as yet is to utilize DEM and compute the local stress tensor from grain fluctuations and inter-grain contact forces per equation 14.

Rycroft et al. [76] have performed DEM simulations of wide silo drainage. The computed stress and flow fields shall now be compared directly against the predictions of the elasto-plastic model. A schematic diagram of the flow geometry is pictured in figure 6(a). In accordance with Rycroft's simulations, we model the silo as having an opening width of $6 d$, a height of $70 d$, and a full width of $150 d$. Rycroft enforced periodic boundary conditions in the $z$ direction giving the silo an apparent $z$ thickness of $8 d$ but without wall-ordering effects. Since the flow should not fluctuate in the $z$ direction, we simply enforce plane-strain conditions (using plane-strain elements of type CPE4R). Furthermore, the silo has left-right symmetry about the vertical center-line, which we take advantage of by modeling only the right half of the silo.

The floor of the silo is modeled as having a frictional interaction with the material characterized by a coefficient of friction $\mu_{\text {floor }}=0.2$. This number was estimated loosely from Rycroft's 

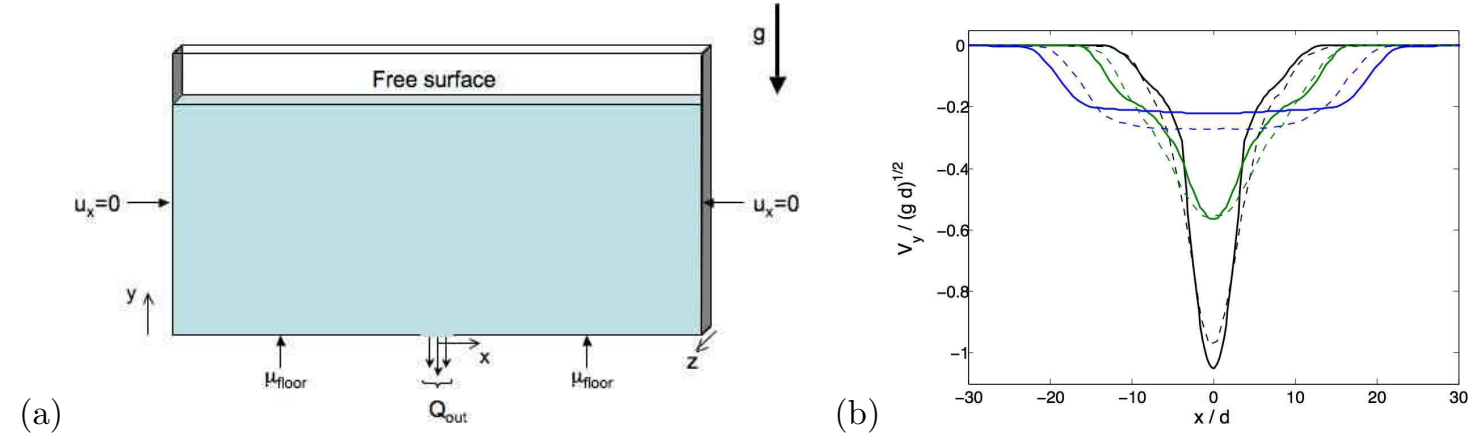

(c)

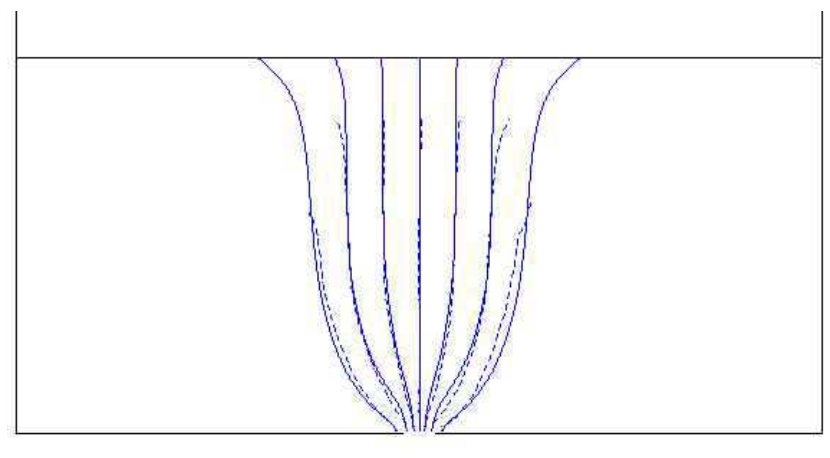

Figure 6: (a) The flat-bottomed silo setup. In (b)-(c), a comparison of elasto-plastic velocity results (-) to DEM data (- -) c/o Rycroft 76]. (b) The $y$ velocity component as a function of $x$ at heights $y=5 d, 10 d, 30 d$. (c) Trajectories predicted by the elasto-plastic model alongside the DEM trajectories. (Container outline provided for ease of viewing.)

simulation - to account for the interaction induced by the effects of particle rolling, rearranging, and dragging along the floor, the element/floor interaction was modeled by reducing Rycroft's floor/particle friction coefficient by $60 \%$. Future study would be necessary to determine the complete and precise form for element/floor sliding interactions. While Rycroft's simulation utilized side walls made of the same frictional material as the floor, for simplicity, the elasto-plastic simulation employs the simpler condition of no $x$ displacement at the side-walls. This could be enhanced in the future, but in wide silos, the details of the side-walls have only a small effect on the dominating behavior.

Ideally speaking, the boundary condition at the silo orifice should be zero stress tractions. However, this is highly problematic numerically. In reality, silo flows develop a "free-fall arch" directly above the orifice [63]. The arch is typically only a few particles high and connects the edges of the opening. Once a particle passes through this hypothetical arch, it enters free-fall and becomes gas-like. A granular material element within the free-fall arch would realistically have a smaller packing fraction, but still support compressive stresses through internal random particle collisions. The elasto-plastic model, however, does not include gas-like effects. Such dilation would be assigned to the elastic deformation, causing the elastic moduli to vanish.

Our interest is not in the details within the immediate vicinity of the orifice, rather the bulk material behavior within the greater silo apparatus. However, with a zero-traction condition at the opening, the situation described above destabilizes the simulation prematurely. While free surfaces are usually taken care of by adding a slight amount of artificial compression, this remedy will not suffice here because the applied pressure has too much of an effect on the evolution of the outflow rate. 
We are left with the alternative of using kinematic boundary conditions at the orifice. It would be overreaching to assign any particular velocity profile at the orifice. Instead, we fix the total flux out the orifice and let the material response determine the shape of the flow profile. To match the outflux in Rycroft's simulation,

$$
Q_{\text {out }} / 2=\int_{\text {Right half-opening }} v_{y}(x, y=0) d x=2.19 \times 10^{-3} \frac{\mathrm{m}^{2}}{\mathrm{~s}}
$$

was instituted at the orifice, encoded as an equation constraint in ABAQUS.

Not far from the opening, large inhomogeneous deformation occurs at small length-scales, necessitating many small elements to maintain accuracy. A grand total of 9750 elements were used in modeling the half-silo. To minimize discretization error, the orifice was modeled with a half-width of 15 elements. The adjacent silo floor was modeled 60 elements wide. The silo height was modeled with 130 elements. The element width was constant within the orifice, but bias was used along the other boundaries to maintain smooth changes in element sizes throughout. Elements shrink vertically as a sole function of distance from the silo bottom. The element width is uniform at the top surface, but moving downward, elements crowd the center so the floor region has a smooth transition from wider elements near the wall to narrower elements adjacent to the orifice.

Due to the high number of elements and small minimal element size to system size ratio, this flow is too computationally intensive for one one processor. Instead, the 12-node Truesdell cluster of the MIT Solid Mechanics Group was employed to solve the problem in parallel. Using domain-level parallelization, the cluster split the half-silo into 12 spatial regions.

The problem solved was as follows: From $t=0-5 \times 10^{-5} \mathrm{~s}$, gravity is gradually turned on while constraining the nodes along the opening from moving in the $y$ direction in order to model a closed orifice. Then, from $t=0.55-5.00 \times 10^{-4} \mathrm{~s}$, the orifice is gradually opened by ramping up the outflow rate from zero to $Q_{\text {out }} / 2$. The simulation is then left to flow until a total time of $t=10^{-3} \mathrm{~s}$ has been reached.

This environment does not possess a steady-state since it is not an Eulerian boundary-value problem at the free surface. Instead, patternistic behavior eventually occurs, which signifies that transients have finished passing - starting at approximately $t=5 \times 10^{-4} \mathrm{~s}$, the velocity and $d^{p}$ fields appear to fluctuate regularly.

The first direct comparison that should be made is between the elasto-plastic and DEM flow profiles. To represent fully-developed mean behavior, Rycroft's flow data was averaged over 100 frames, during a period of what appears to be transient-free flow. Similarly, the elasto-plastic flow was also averaged over many frames of fully-developed motion. To improve the validity of this average, the model was run an extra $5 \times 10^{-4}$ s longer and the time average was performed over the range $t=0.5-1.5 \times 10^{-4} \mathrm{~s}$, comprising 127 frames. Figure 6 displays the comparison. Overall the agreement is sufficient. The particular way in which the peak in the downward velocity component broadens as height increases is well captured by the model. Once again, as is now a common theme, the elasto-plastic downward velocity appears to change more rapidly in space. Non-local effects such as diffusion could smooth out these sharper variations and possibly improve the agreement. Observing figure 6(c), the DEM and elasto-plastic trajectories agree well, especially below $y \approx 40 d$. The differences in the upper silo trajectories could stem from the fact that in the DEM simulation, the top free surface lowered notably by the time steady flow could be imaged, whereas the elastoplastic simulation reached this point before any noticeable drop of the top free surface (due to the artificial density reduction).

To compare instantaneous behavior, we observe snapshots of the shearing rate profile. As shown in figure 7. well-developed elasto-plastic behavior involves two long "arms" of shearing that extend from the edges of the orifice to the top surface. From those arms, narrower, weaker shear bands (note the log-scale) continually drop down, creating an intersecting "mesh". The DEM solution does look similar, with two dominant arms of shearing. Note as well that near the top of the silo, both plots show the shearing spreads out approaching the free surface. The most obvious difference, 

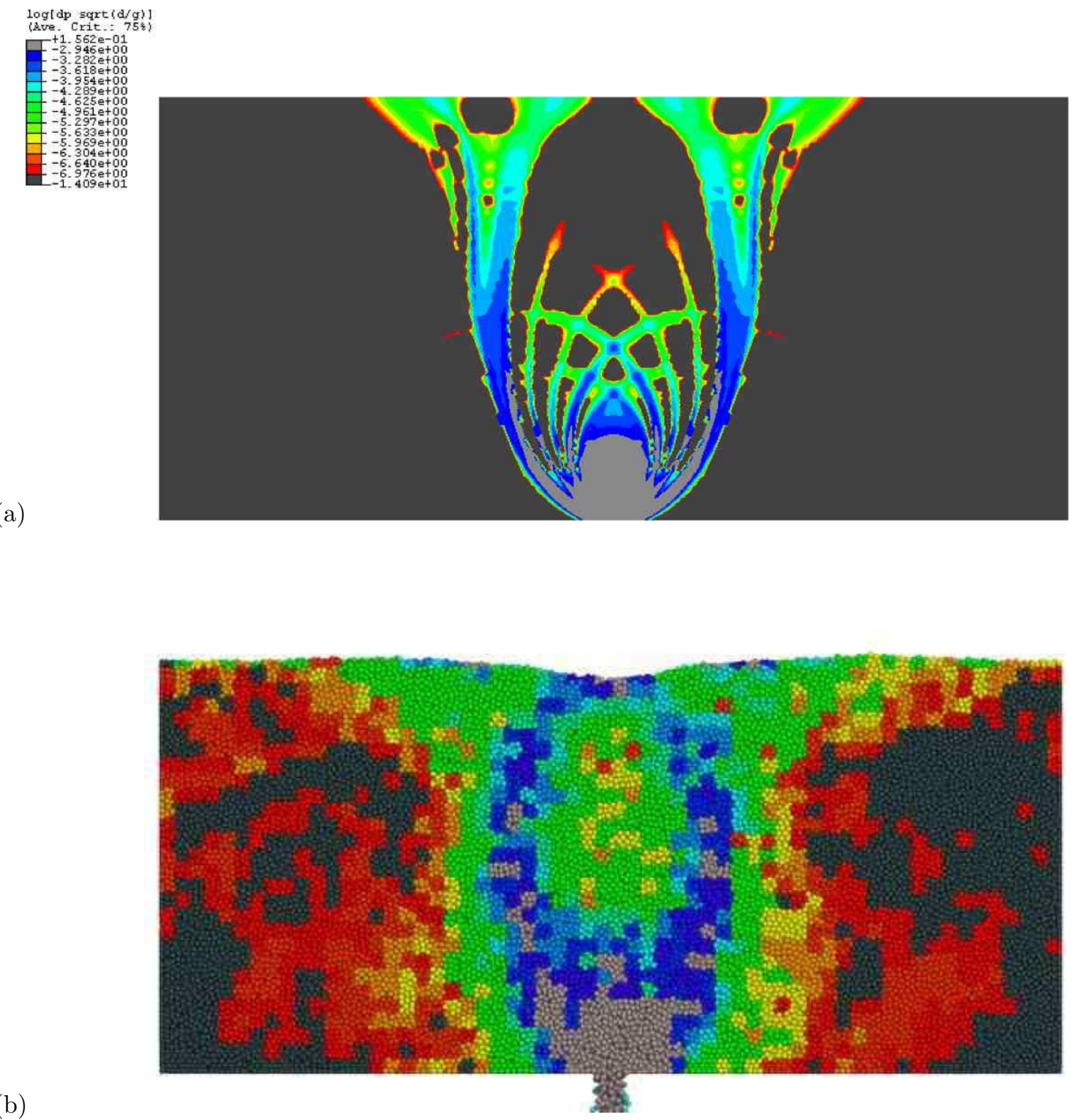

(b)

Figure 7: The plastic shearing rate $d^{p}$, expressed in units of $\sqrt{g / d}$, plotted in logarithm form to accentuate small features. (a) The elasto-plastic solution: Note the intricate pattern of shear bands that fill the region between the two long shearing arms. The long-time behavior has the bands fall down one-by-one from the larger shearing arms. (b) The DEM solution c/o Rycroft [76]: Similar to the elasto-plastic except blurred out by the box-averaging and the effects of non-local "diffusion" that are ignored by first-order elasto-plasticity. Both plots use the same color scale. 


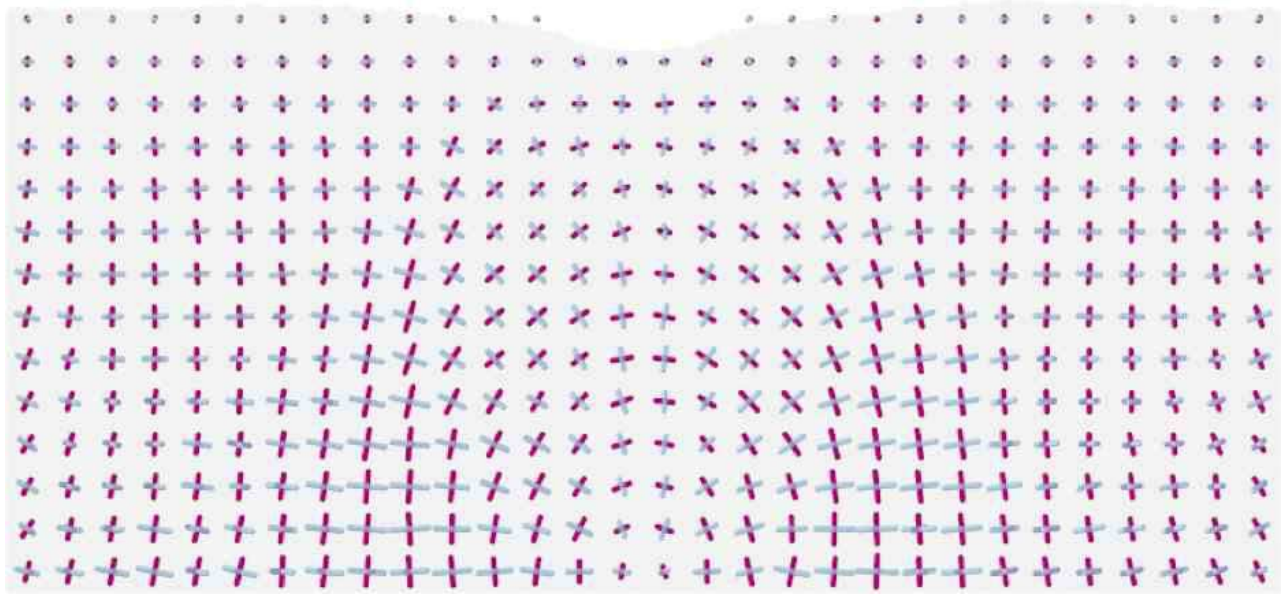

Figure 8: The instantaneous deviatoric principal stress directions plotted as crosshairs with lengths corresponding to the associated deviatoric stress eigenvalues. (a) The elasto-plastic solution: The thicker of the lines corresponds to the major principal stress direction. (b) The DEM solution c/o Rycroft [76]: Major principal stress in purple, minor (and intermediate where visible) in blue. 


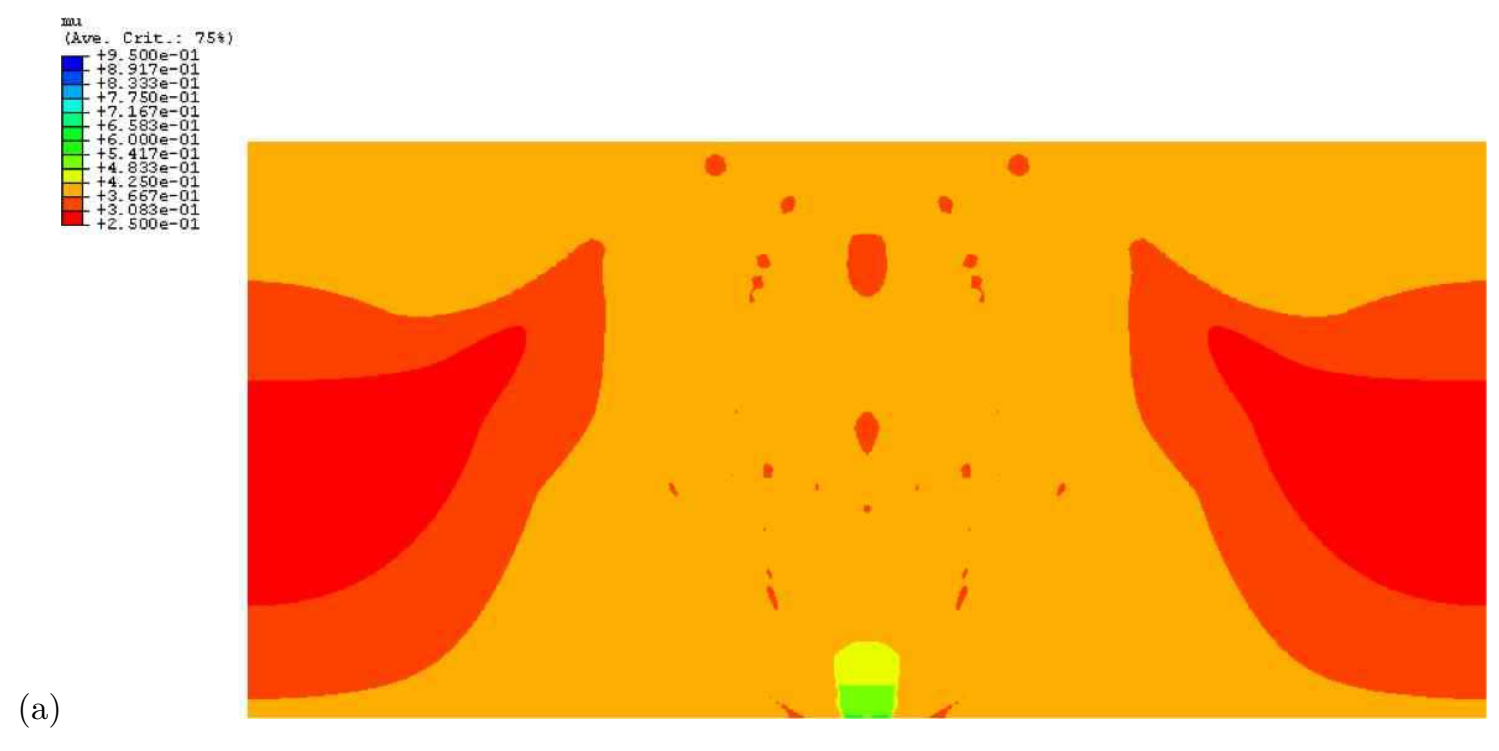

(b)

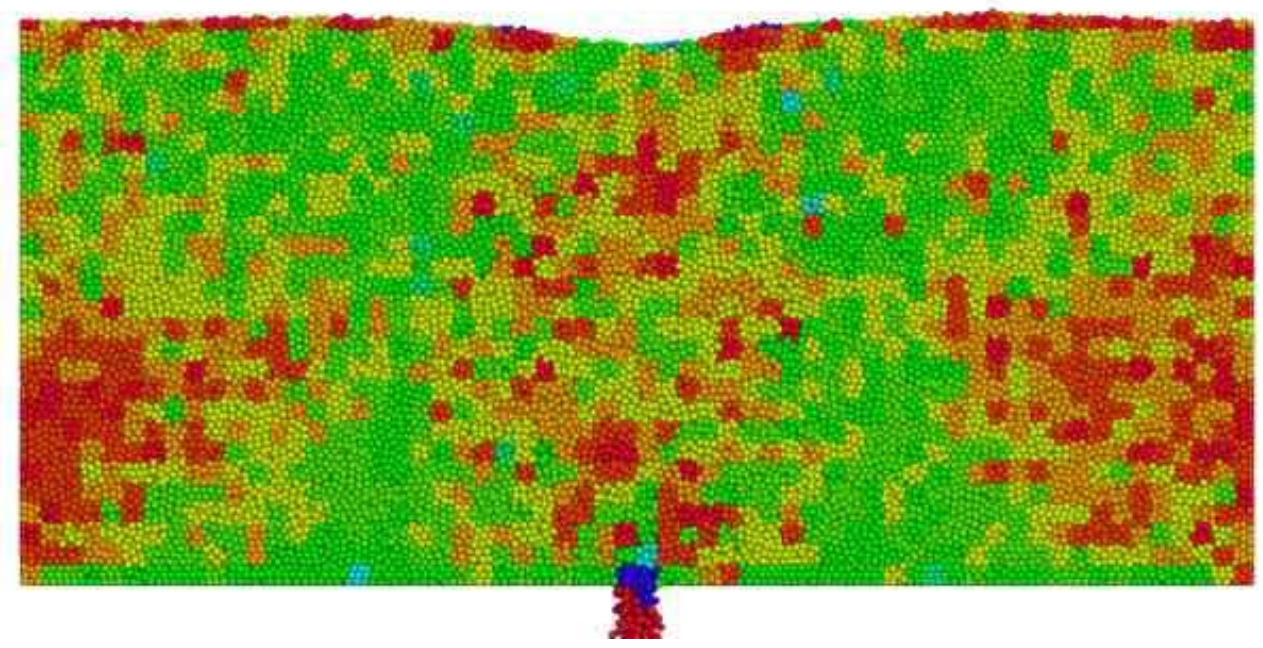

Figure 9: The instantaneous stress ratio $\mu$ during fully developed flow. (a) The elasto-plastic solution. (b) The DEM solution c/o Rycroft [76]. Both plots use the same color scale. 
as has been previously noted, is that the elasto-plastic solution has sharp flow cutoffs whereas the shear rate always gradually tails off in the DEM. To reiterate, the model lacks non-local quasi-static terms so flow cutoffs are to be expected. Even if narrow bands did exist in the DEM, the box-average being performed would most assuredly obscure them.

Moving on to the stresses, we first check the principal stresses and directions during fullydeveloped flow. As is evident in figure 8, the principal stress orientations predicted by the elastoplastic model closely match those of the DEM. Both show the major principal stresses forming "arches" about the orifice and, moving away from the opening, the principal stress chains transition to becoming more vertical. In the DEM, it is apparent that the major principal stresses adjacent to the side walls have a slightly tilted orientation, whereas those of the elasto-plastic solution remain almost perfectly vertical. This is entirely due to the chosen boundary conditions. The DEM utilized frictional sidewalls and the tilt indicates the walls are exerting an upward shear on the material. This wall shear can be traced back to the filling process, where pouring causes an "active wall state" that resists the downward motion of the grains. On the contrary, the side walls in the elasto-plastic simulation are frictionless. A side wall friction may be included in the future to better model this minor effect.

For a more direct comparison of the relative sizes of the stress invariants, see figure 9, Qualitatively, the two solutions show similar spatial changes. Both show a region of lower $\mu$ that swoops up from the lower side walls together with scattered minima of $\mu$ in the upper-middle region of the silo. Quantitatively, it appears the $\mu$ values in the flowing zone are lower for the elasto-plastic solution. A probable explanation for this could be that the grains in the DEM simulation have a different surface roughness than the glass beads of [43] and should in actuality have higher $\mu_{s}$ and $\mu_{2}$ values. Recall that our material parameters were not extracted from the DEM data, but come from two different papers. In [17] it was demonstrated that increasing the surface roughness of grains causes $\mu_{s}$ to increase. If the elasto-plastic model were implemented with a larger friction constants, the $\mu$ profile would indeed increase in the flowing regions, as larger $\mu$ would be needed to invoke the same plastic shear rate.

\section{Conclusion and future directions}

This work has demonstrated a highly general, 3D granular continuum model that unifies the recent results in granular elasticity and plasticity. The unification follows a rigorous $\mathbf{F}^{e} \mathbf{F}^{p}$ decomposition, with elastic and plastic response combined using finite-deformation elasto-plasticity. The model can be used to predict flows uniquely in any environment with mechanically well-posed boundary conditions and/or body forces. The model was implemented as a user-material in ABAQUS/Explicit and tested in three unrelated geometries. With no fitting, it appears to give qualitative, and in some case quantitative, predictions for both the stress and velocity field in arbitrary granular flow geometries. Even so, there are a few clear avenues of future work with regard to improving the current model.

\subsection{Quasi-static non-locality}

The most glaring effect absent from the model is that it cannot account for "blurring" in the flow fields. As described in depth in sections 4.2 and 4.3 when the normalized shear rate decreases low enough, the rheology is no longer determinable from a simple relation of the form $I=g^{-1}(\mu)$.

One would speculate a more complete form has higher order gradients in stress, flow rate, and/or state parameters balanced by some additional grain-level length-scale. In regions of moderate flow rate, these spatially second order effects should be dominated by the local rheology. This is clear from the above tests, which show the model does indeed perform better where the flow is faster. But equally clear is the fact that these terms cannot be ignored near static yield. Section 4.3 lists several possible theories to describe non-local behavior, but how each candidate would fit theoretically within the current model remains to be studied. 
By neglecting quasi-static non-locality, the model is tacitly assuming that the material is capable of forming clean solid-like/fluid-like interfaces. This is almost always an idealization that is unrealized, as particles in a random packing rarely have the geometric ability to assemble in a fashion that avoids overlap with the predicted interface. A particle on the interface, being unable to both shear with the fluid and remain static with the solid, instead transmits some of the shearing behavior from the fluid-like zone into the solid zone, thereby explaining the gradual tails we see in actual granular flow profiles. Under particular circumstances, however, granular flow can be made to segregate clearly into flowing and completely static zones. Thompson and Grest [83] have shown that a monodisperse 2D disk assembly undergoing horizontal planar shear with downward gravity does indeed have a flow cutoff with zero flow occurring beneath a shear band at the top. This behavior is a rarity brought on by the fact that solid-like material in this geometry can and does form a hexagonal crystal and the horizontal fluid/solid interface happens to align perfectly with a crystal plane.

\subsection{Dilation}

The present model avoids all inelastic dilation. Though an argument for why this is acceptable for our current purposes is presented in section 4.4 certain benefits would come with properly accounting for the small amount of dilation that occurs in dense flow.

Inelastic dilation comes in two primary forms: plastic and gas-like. Shear and shearing dilation, as described in section 4.2, are prototypical plastic mechanisms. Gas-like dilation can occur in particularly energetic surroundings, even below the plastic yield criterion - for example, consider shaking a box of rubber spheres under uniform pressure. Granted, gas-like behavior is outside the dense flow regime we currently study. Even so, it is possible for a small region of an otherwise dense flow to become gas-like, and consequently a means of dealing with this behavior is desired. Recall this was a crucial issue in the silo geometry, which is almost entirely dense except within the small free-fall arch that encompasses the opening. Gas-like effects may require a granular temperature and heat flux to express the internal pressure.

Several models for plastic dilation have been proposed (see section 4.4), but a direct 3D discrete element study would be ideal for quantifying the precise dilatational dependences. One might hypothesize, based on equation 5 , that the plastic dilation has a form

$$
\frac{d \eta}{d \gamma^{p}}=\frac{\dot{\eta}}{\tilde{d}^{p}(\mathbf{M})}=A(\eta) B\left(\Phi(I)-0.63 e^{-\eta}\right)
$$

where $\eta=\log \left(\operatorname{det} \mathbf{F}^{p}\right)$ measures plastic dilation, $\gamma^{p}$ is a plastic shear strain, $I=\tilde{d}^{p}(\mathbf{M}) /\left(\frac{1}{d} \sqrt{\frac{P}{\rho_{s}}}\right)$ is the inertial number, and the functions $A$ and $B$ are empirical, with $B=1$ when the magnitude of its argument is large and $B \rightarrow 0$ as the input goes to zero. The above states that a material originally at random close packing $(\Phi=0.63)$ obeys Bagnold type dependences at steady flow. While the flow is unsteady, it dilates according to $A$ and then ceases dilation as the Bagnold relationship is approached. A relation of this form appears to agree with results of Rycroft et al. 76], and collaborative efforts with Rycroft are underway to quantify this evolution law.

Once a form for the plastic dilation has been verified, it would serve to enhance the computation of elasto-statics, where the moduli are known to vary with the packing fraction, as well as give meaningful packing fraction data throughout. However, the technique of artificial density reduction may no longer be valid, since the steady packing fraction, while only a few percent different than at the start, may take more time to develop than is typically allotted in a simulation run.

\subsection{Flow condition}

The plastic flow rule of Jop et al. asserts the codirectionality condition. A direct test of the flow condition should be performed to verify whether this is indeed the best candidate. Such a test may be forthcoming with recent DEM data on large conical hopper flow. Some models have 
had success utilizing double-shearing conditions [1], and Rycroft's silo flow data has shown some deviation from codirectional flow. Even so, codirectionality has fit the current needs and appears to be sufficient to predict basic flow behavior. A fine-tuned flow condition would be essential to model highly asymmetric 3D flows.

\section{Acknowledgements}

The author gratefully acknowledges Lallit Anand and the MIT Solid Mechanics Group for helpful discussion and access to the ABAQUS software package. The author also thanks Chris H. Rycroft

for access to discrete simulation data and acknowledges Martin Z. Bazant for advice. This work was supported by the NDSEG and NSF GRFP fellowship programs.

\section{References}

[1] L. Anand and C. Gu. Granular materials: constitutive equations and strain localization. $J$. Mech. Phys. Solids, 28:1701, 2000.

[2] L. Anand and C. Su. A theory for amorphous viscoplastic materials undergoing finite deformations, with application to metallic glasses. J. Mech. Phys. Solids, 53:1362-1396, 2005.

[3] I. S. Aranson and L. S. Tsimring. Continuum description of avalanches in granular media. Phys. Rev. E, 64:020301, 2001.

[4] I. S. Aranson and L. S. Tsimring. Continuum theory of partially fluidized granular flows. Phys. Rev. E, 65:061303, 2002.

[5] I. S. Aranson and L. S. Tsimring. Patterns and collective behavior in granular media: Theoretical concepts. Rev. Mod. Phys., 78:641-692, 2006.

[6] R. A. Bagnold. Experiments on a gravity free dispersion of large solid spheres in a newtonian fluid under shear. Proc. Roy. Soc. London Ser. A, 225, 1954.

[7] N.J. Balmforth and A. Provenzale. Patterns of dirt. Geomorph. Fluid Mech., 582:164-187, 2001.

[8] M. Z. Bazant. The spot model for random-packing dynamics. Mechanics of Materials, 38:717$731,2006$.

[9] R. P. Behringer. Contact force measurements and stress-induced anisotropy in granular materials. Nature, 435:1079-1082, 2005.

[10] L. Bocquet, W. Losert, D. Schalk, T. C. Lubensky, and J. P. Gollub. Granular shear flow dynamics and forces: Experiment and continuum theory. Phys. Rev. E, 65:011307, 2002.

[11] J.-P. Bouchaud, M. E. Cates, and P. Claudin. Stress distribution in granular media and nonlinear wave equation. J. Phys., 5:639-656, 1995.

[12] J.-P. Bouchaud, M. E. Cates, J. R. Prakash, and S. F. Edwards. A model for the dynamics of sandpile surfaces. J. Phys. I (France), 4:1383, 1994.

[13] J.-P. Bouchaud, M. E. Cates, J. R. Prakash, and S. F. Edwards. Hysteresis and metastability in a continuum sandpile model. Phys. Rev. Lett., 74:1982, 1995.

[14] T. Boutreux, H. A. Makse, and P.-G. de Gennes. Surface flows of granular mixtures. Euro. Phys. J. B, 9:105-115, 1999. 
[15] T. Boutreux, E. Raphaël, and P.-G. de Gennes. Surface flows of granular materials: A modified picture for thick avalanches. Phys. Rev. E, 58:4692-4700, 1998.

[16] J. Choi, A. Kudrolli, and M. Z. Bazant. Velocity profile of gravity-driven dense granular flow. J. Phys.: Condensed Matter, 17:S2533-S2548, 2005.

[17] F. da Cruz, S. Emam, M. Prochnow, J. Roux, and F. Chevoir. Rheophysics of dense granular materials: Discrete simulation of plane shear flows. Phys. Rev. E., 72:021309, 2005.

[18] P. G. de Gennes. Granular matter: a tentative view. Rev. Mod. Phys., 71:S374-S382, 1999.

[19] M. Depken, J. B. Lechman, M. van Hecke, W. van Saarloos, and G. S. Grest. Stresses in smooth flows of dense granular media. Europhys. Lett., 78:58001, 2007.

[20] P. J. Digby. The effective elastic modulus of porous granular rocks. J. Appl. Mech., 48:803, 1981.

[21] A. Drescher. Analytical Methods in Bin-Load Analysis. Elsevier, 1991.

[22] J. Duffy and R. D. Mindlin. Stress-strain relation and vibrations of granular medium. J. Appl. Mech., 24:585, 1957.

[23] S. F. Edwards. In Blackman and Taguena, editors, Disorder in Condensed Matter Physics. Oxford, 1991.

[24] S. F. Edwards and D. V. Grinev. Granular media as a physics problem. Advances in Complex Systems, 4:451-467, 2001. (also reprinted in Ref. [36]).

[25] D. Ertaş and T. C. Halsey. Granular gravitational collapse and chute flow. Europhys. Lett., 60:931-937, 2002.

[26] P. Evesque and P. G. de Gennes. On the statics of silos. C. R. Acad. Sci. (PARIS), Ser. II, 326:761, 1998.

[27] M. L. Falk and J. S. Langer. Dynamics of viscoplastic deformation in amorphous solids. Phys. Rev. E, 57:7192-7205, 1998.

[28] J. Geng, D. Howell, E. Longhi, R. P. Behringer, G. Reydellet, L. Vanel, E. Clèment, and S. Luding. Footprints in sand: The response of a granular material to local perturbations. Phys. Rev. Lett., 87:035506, 2001.

[29] B. J. Glasser and I. Goldhirsch. Scale dependence, correlations, and fluctuations of stresses in rapid granular flows. Phys. Fluids, 13:407, 2001.

[30] J. D. Goddard. Nonlinear elasticity and pressure-dependent wave speeds in granular media. Proc. R. Soc. London, Ser. A, 430:105, 1990.

[31] C. Goldenberg, A. P. F. Atman, P. Claudin, G. Combe, and I. Goldhirsch. Scale separation in granular packings: Stress plateaus and fluctuations. Phys. Rev. Lett., 96:168001, 2006.

[32] C. Goldenberg and I. Goldhirsch. Friction enhances elasticity in granular solids. Nature, 435:188-191, 2005.

[33] M. E. Gurtin and L. Anand. A theory of strain-gradient plasticity for isotropic, plastically irrotational materials. part i: Small deformations. J. Mech. Phys. Solids, 53:1642-1649, 2005.

[34] M. E. Gurtin and L. Anand. A theory of strain-gradient plasticity for isotropic, plastically irrotational materials. part ii: Finite deformations. J. Mech. Phys. Solids, 21:2297-2318, 2005. 
[35] Morton E. Gurtin. An Introduction to Continuum Mechanics. Academic Press, 1981.

[36] T. Halsey and A. Mehta, editors. Challenges in Granular Physics. World Scientific, 2002.

[37] K. Hashiguchi and S. Tsutsumi. Gradient plasticity with the tangential-subloading surface model and the prediction of shear-band thickness of granular materials. Int. J. Plasticity, 23:767-797, 2007.

[38] O. Al Hattamleh, B. Muhunthan, and H. M. Zbib. Gradient plasticity modelling of strain localization in granular materials. Int. J. Numer. Anal. Meth. Geomech., 28:465-481, 2004.

[39] R. Hill. The Mathematical Theory of Plasticity. Oxford at the Clarendon Press, 1950.

[40] I. Iordanoff and M. M. Khonsari. Granular lubrication: toward an understanding between kinetic and fluid regime. ASME J. Tribol., 126:137-145.

[41] H. M. Jaeger, S. R. Nagel, and R. P. Behringer. Granular solids, liquids, and gases. Rev. Mod. Phys., 68:1259-1273, 1996.

[42] Y. Jiang and M. Liu. Granular elasticity without the coulomb condition. Phys. Rev. Lett., 91:144301, 2003.

[43] P. Jop, Y. Forterre, and O. Pouliquen. Crucial role of side walls for granular surface flows: consequences for the rheology. J. Fluid, Mech., 541:21-50, 2005.

[44] P. Jop, Y. Forterre, and O. Pouliquen. A constitutive law for dense granular flows. Nature, 441:727, 2006.

[45] L. P. Kadanoff. Built upon sand: Theoretical ideas inspired by the flow of granular materials. Rev. Mod. Phys., 71:435-444, 1999.

[46] K. Kamrin. Stochastic and Deterministic Models for Dense Granular Flow. PhD thesis, Massachusetts Institute of Technology, 2008.

[47] K. Kamrin and M. Z. Bazant. Stochastic flow rule for granular materials. Phys. Rev. E, 75:041301, 2007.

[48] K. Kamrin, C. H. Rycroft, and M. Z. Bazant. The stochastic flow rule: A multi-scale model for granular plasticity. Modelling Simul. Mater. Sci. Eng., 15:S449, 2007.

[49] Georg Koval. Compotement d'interface des matériaux granulaires. PhD thesis, École Nationale des Ponts et Chaussées, 2008.

[50] E. Kröner. Allgemeine kontinuumstheorie der versetzungen und eigenspannungen. Arch. Ration. Mech. Anal., 4:273-334, 1960.

[51] R. Kuwano and R. J. Jardine. On the applicability of cross-anisotropic elasticity to granular materials at very small strains. Géotechnique, 52:10:727-749, 2002.

[52] E. H. Lee. Elastic plastic deformation at finite strain. J. Appl. Mech., 36:1-6, 1969.

[53] Anaël Lemaître. Origin of a repose angle: Kinetics of rearrangements for granular materials. Phys. Rev. Lett., 89:064303, 2002.

[54] Anaël Lemaître. Rearrangements and dilatency for sheared dense materials. Phys. Rev. Lett., 89:195503, 2002.

[55] J. Litwiniszyn. Statistical methods in the mechanics of granular bodies. Rheol. Acta, 2/3:146, 1958. 
[56] G. Lois, A. Lemaitre, and J. M. Carlson. Numerical tests of constitutive laws for dense granular flows. Phys. Rev. E., 72:051303, 2005.

[57] W. Losert, L. Bocquet, T. C. Lubensky, and J. P. Gollub. Particle dynamics in sheared granular matter. Phys. Rev. Lett., 85:1428-1431, 2000.

[58] H. A. Makse, N. Gland, D. L. Johnson, and L. Schwartz. Granular packings: Nonlinear elasticity, sound propagation, and collective relaxation dynamics. Phys. Rev. E, 70:061302, 2004.

[59] G. D. R. Midi. On dense granular flows. Euro. Phys. Journ. E., 14:341-365, 2004.

[60] P. Mills, D. Loggia, and M. Tixier. Model for a stationary dense granular flow along an inclined wall. Europhys. Lett., 45:733-738, 1999.

[61] R. D. Mindlin and H. Deresiewicz. Elastic spheres in contact under varying oblique forces. $J$. Appl. Mech., 20:327-244, 1953.

[62] J. Mullins. Stochastic theory of particle flow under gravity. J. Appl. Phys., 43:665, 1972.

[63] R. M. Nedderman. Statics and Kinematics of Granular Materials. Cambridge University Press, 1992.

[64] R. M. Nedderman and U. Tüzün. Kinematic model for the flow of granular materials. Powder Technology, 22:243, 1979.

[65] A. N. Norris and D. L. Johnson. Nonlinear elasticity of granular media. J. Appl. Mech., 64:39, 1997.

[66] M. Ostoja-Starzewski. Material spatial randomness: From statistical to representative volume element. Prob. Eng. Mech., 21:112-132.

[67] M. Ostoja-Starzewski. Scale effects in plasticity of random media: status and challenges. Int. J. Plasticity, 21:1119-1160, 2005.

[68] O. Pouliquen. Scaling laws in granular flows down rough inclined planes. Phys. Fluids, 11:542, 1999.

[69] O. Pouliquen, Y. Forterre, and S. Le Dizes. Slow dense granular flows as a self-induced process. Advances in Complex Systems, 4:441-450, 2001. (also reprinted in Ref. [36]).

[70] O. Pouliquen and R. Gutfraind. Stress fluctuations and shear zones in quasistatic granular chute flows. Phys. Rev. E, 53:552-561, 1996.

[71] M. Prochnow. Ecoulemnets denses de grains secs. PhD thesis, Ecole Nationale de Ponts et Chaussées, Marne la Vallée, France, 2002.

[72] L. La Ragione, V. C. Prantil, and I. Sharma. A simplified model for inelastic behavior of an idealized granular material. Int. J. Plasticity, 24:168-189, 2008.

[73] J. W. Rudnicki and J. R. Rice. Conditions for the localization of deformation in pressuresensitive and dilatant materials. J. Mech. Phys. Solids, 23:371, 1975.

[74] C. H. Rycroft. private communication.

[75] C. H. Rycroft, M. Z. Bazant, G. S. Grest, and J. W. Landry. Dynamics of random packings in granular flow. Physical Review E, 73:051306, 2006. 
[76] C. H. Rycroft, K. Kamrin, and M. Z. Bazant. Assessing continuum hypotheses in simulation of granular flow. In press, DOI: 10.1016/j.jmps.2009.01.009, 2009.

[77] A. Samadani, A. Pradhan, and A. Kudrolli. Size segregation of granular matter in silo discharges. Phys. Rev. E, 60:7203-7209, 1999.

[78] S. B. Savage. Analyses of slow high-concentration flows of granular materials. J. Fluid Mech., $377: 1,1998$.

[79] A. Schoefield and P. Wroth. Critical State Soil Mechanics. McGraw-Hill, 1968.

[80] L. E. Silbert, D. Ertas, G. S. Grest, T. C. Halsey, D. Levine, and S. J. Plimpton. Granular flow down an inclined plane: Bagnold scaling and rheology. Phys. Rev. E., 64:051302, 2001.

[81] V. V. Sokolovskii. Statics of Granular Materials. Pergamon/Oxford, 1965.

[82] A. J. M. Spencer. A theory of the kinematics of ideal soils under plane strain conditions. $J$. Mech. Physics, 12:337351, 1964.

[83] P. A. Thompson and G. S. Grest. Granular flow - friction and the dilatancy transition. Phys Rev Lett, 67:1751-1754, 1991.

[84] C. Thornton and L. Zhang. A numerical examination of shear banding and simple shear noncoaxial flow rules. Phil. Mag., 86:3425-3452, 2006.

[85] J.-C. Tsai, G. A. Voth, and J. P. Gollub. Internal granular dynamics, shear-induced crystallization, and compaction steps. Phys. Rev. Lett., 91:064301, 2003.

[86] S. Tsutsumi and K. Hashiguchi. General non-proportional loading behavior of soils. Int. J. Plasticity, 21:1941-1969, 2005.

[87] S. Tsutsumi and K. Kaneko. Constitutive response of idealized granular media under the principal stress axes rotation. Int. J. Plasticity, 24:1967-1989, 2008.

[88] I. Vardoulakis and E. C. Aifantis. A gradient flow theory of plasticity for granular media. Acta Mechanica, 87:197-217, 1991.

[89] K. Walton. The effective elastic moduli of a random packing of spheres. J. Mech. Phys. Solids, $35: 213,1987$.

[90] H. Zhu, M. M. Mehrabadi, and M. Massoudi. Three-dimensional constitutive relations for granular materials based on the dilatant double shearing mechanism and the concept of fabric. Int. J. Plasticity, 22:826-857, 2006. 